\title{
Single and double helix vortex breakdown as two dominant global modes in turbulent swirling jet flow
}

\author{
Maarten Vanierschot ${ }^{1}$, Jens S. Müller ${ }^{2}$, Moritz Sieber ${ }^{2}$, Mustafa Percin ${ }^{3}$, Bas W. van \\ Oudheusden $^{4}$, and Kilian Oberleithner ${ }^{2}$ \\ ${ }^{1}$ Mechanical Engineering Technology Cluster TC, Group T Leuven campus, KU Leuven, \\ Leuven, Belgium \\ ${ }^{2}$ Laboratory for Flow Instabilities and Dynamics, Institut für Strömungsmechanik und \\ Technische Akustik, Technische Universität Berlin, Berlin, Germany \\ ${ }^{3}$ Department of Aerospace Engineering, Middle East Technical University, Ankara, Turkey \\ ${ }^{3}$ Department of Aerospace Engineering, TU Delft, Delft, The Netherlands
}

November 28, 2019

\begin{abstract}
In this paper, we study the shape and dynamics of helical coherent structures found in the flow field of an annular swirling jet undergoing vortex breakdown. The flow field is studied by means of time-resolved tomographic particle image velocimetry measurements. The obtained flow fields are analysed using both classical and spectral proper orthogonal decomposition (POD and SPOD). Despite the simple geometrical setup of the annular jet, the flow field is very complex. Two distinct large-scale helical flow structures are identified: a single and a double helix, both co-rotating with the swirl direction and it is revealed that these structures are not higher harmonics of each other. The structures have a relatively low energy content which makes it hard to separate them from other dynamics of the flow field, notably turbulent motions. Because of this, classic POD fails to identify both structures properly. SPOD, on the other hand, allows them to be identified accurately when the filter size is set at around 8 times the precession period. The precession frequencies of the single and double helix correspond to Strouhal numbers of 0.273 and $0.536 \pm 0.005$, respectively. A global stability analysis of the mean flow field shows that these structures correspond to two separate global modes. The precessing frequencies obtained by the stability analysis and the related spatial structures match very well with the experimental observations. The current work extends our knowledge on turbulent vortex breakdown and on mean field global stability theory in general. It leads to the following conclusions. Firstly, single and double helix vortex breakdown are both manifestations of global modes. Previous studies have shown that both $m=1$ and $m=2$ modes can coexist in swirling jets. However, the $m$ $=2$ mode has been identified as a second harmonic of the first mode, while this study identifies both as two independent global modes. Secondly, this work shows that the simultaneous occurrence of multiple helical global modes is possible within a turbulent flow and their shapes and frequencies are very well predicted by mean field stability analysis. The latter finding is of general interest as it applies to a wide class of fluid problems dominated by multiple oscillatory structures.
\end{abstract}

\section{Introduction}

It is well known that swirling flows can sustain large-scale coherent structures, such as vortex breakdown and the Precessing Vortex Core (PVC), which can be found in both laminar or turbulent flow regimes. A comprehensive overview of these structures and their occurrence can be found in the reviews of Lucca-Negro \& O'Doherty (2001) and Syred (2006). Experimental studies show that vortex breakdown can be classified in no less than seven different types. The two most commonly observed, called bubble and spiral breakdown, were first reported by Lambourne \& Bryer (1961). In bubble breakdown, an axisymmetric region of flow recirculation is formed near the central axis of rotation, acting as a bluff body in the flow. In spiral breakdown, the vortex core breaks up into a helical structure, which precesses around the central axis. Up till now, due to the occurrence of several types of breakdown and the often contradictory results because of a high sensitivity to boundary conditions, there is still no general consensus about the origin of vortex breakdown. Recent studies appear to support the criterion of Benjamin (Benjamin, 1962), which states that vortex breakdown is the transition from a supercritical to a subcritical flow. If this criterion is applied locally at a certain axial location in the jet, it can predict the region of bubble appearance (Ruith et al., 2003; Oberleithner et al., 2012). This has been confirmed by the recent simulations of Vanierschot (2017) who showed that in a flow region near the central axis, which is 
subcritical according to Benjamin's definition, an axisymmetric imbalance in the momentum equation grows exponentially, which eventually leads to the formation of the vortex breakdown bubble. On the other hand, spiral vortex breakdown or the PVC does not originate from axisymmetric instabilities, but from helical ones. For low Reynolds numbers, many studies have shown that spiral breakdown is the manifestation of a global mode of the flow which develops in the periphery of the breakdown bubble (Liang \& Maxworthy, 2005; Ruith et al., 2003; Gallaire et al., 2006a; Oberleithner et al., 2011; Meliga et al., 2012a; Qadri et al., 2013; Rukes et al., 2017). Two modes of spiral breakdown have been observed: the single helix $(|m|=1)$ and the double helix $(|m|=2)$, where $m$ is the azimuthal wavenumber. The recent study of Meliga et al. (2012a) shows that both single or double helix breakdown are bifurcations from axisymmetric breakdown and that mode selection depends on the swirl number. In experimental studies, the observation of the double helical mode is very rare (Sarpkaya, 1971; Escudier \& Zehnder, 1982; Billant et al., 1998; Gallaire et al., 2004) as this type of breakdown is highly sensitive to disturbances. It is mostly observed in pipe flows, however it has also been reported to occur in the flow over a delta wing at large incidence angles (Calderon et al., 2012) or in annular jet flows (Vanierschot et al., 2018).

The annular jet is a special kind of jet flow, i.e. one compromising a round channel with a parallel centerbody in the middle. This jet configuration is widely used in bluff-body combustors. The specific appearance of a central bluff-body creates a central recirculation zone (CRZ) which enhances mixing and flame stabilisation (Beér \& Chigier, 1983; Gupta \& Lilley, 1984). Moreover, it can be used as a fuel injection device or to induce a coflow jet in the center, e.g. in the Sydney burner (Dinesh \& Kirkpatrick, 2009; Dinesh et al., 2012). The flow field of the annular jet can be divided into three zones: the initial merging, the intermediate and the fully merged zone (Ko \& Chan, 1979). The initial merging zone contains the wake behind the centerbody, and extends up to the end of the potential core of the jet. In this zone, two shear layers exist: an inner shear layer between the jet and the CRZ and an outer shear layer between the jet and the surroundings. Both shear layers are prone to Kelvin-Helmholtz instabilities which are convected downstream. For the inner shear layer, the instabilities are convected back to the jet nozzle due to recirculation, creating a very low frequency meandering of the wake (Vanierschot et al., 2014). As such, the position of the stagnation point at the end of the wake exhibits a dynamic behavior and velocity measurements show large fluctuations in that region (Durao \& Whitelaw, 1978; Patte-Rouland et al., 2001; Danlos \& Patte-Rouland, 2013). This low frequency meandering can also be found in other types of wake flows, such as behind Ahmed bodies (Lucas et al., 2017; Pavia et al., 2018), blunt bodies (Grandemange et al., 2013; Brackston et al., 2016), axisymmetric wakes (Rigas et al., 2014, 2015) or axisymmetric afterbodies (Gentille et al., 2016, 2017). Adding swirl to an annular jet induces the same favorable effects as the wake behind the inner body, i.e. the creation of regions of intensified mixing and low flow velocity. For instance, in combustion, swirl is often added to the jet to enhance flame stabilisation. The addition of swirl has a significant impact on the flow structures in the initial merging region of the annular jet. If a very small amount of swirl is added, the Kelvin-Helmholtz instability in the inner and outer shear layers becomes helical (Garcia-Villalba et al., 2006; Garcia-Villalba \& Frohlich, 2006; Jones et al., 2012; Zhang et al., 2015) and the meandering of the wake at zero swirl transforms into a precession (Vanierschot \& Van den Bulck, 2011; Vanierschot et al., 2014). As the level of swirl is further increased, these helical instabilities become stronger. At some point in the swirl increase, the central recirculation zone behind the centerbody opens and a toroidal vortex is formed (Sheen et al., 1996; Vanierschot \& Van den Bulck, 2008). When the critical swirl number is reached, a recirculation zone appears downstream, called the vortex breakdown bubble. With further increase in swirl, this bubble moves upstream and interacts with the wake behind the bluff body, creating a complex flow field. After merging of the CRZ and the vortex breakdown bubble, the coherent structures in the flow are very similar to the ones observed in round jet flows. Like for round jets, the PVC is reported as a single helix which is wrapped around the breakdown bubble (Sheen et al., 1996; Huang et al., 2003; Huang \& Yang, 2005; Garcia-Villalba et al., 2006; Garcia-Villalba \& Frohlich, 2006; Dinesh \& Kirkpatrick, 2009; O'Connor \& Lieuwen, 2012; Stoehr et al., 2012; Falese et al., 2014; Canepa et al., 2015; Poinsot, 2017).

Local linear stability analysis (LSA) has been proven to predict the PVC with regard to temporal frequency and spatial mode shape (Chomaz, 2005) in both laminar (Gallaire et al., 2006b) and turbulent flows (Oberleithner et al., 2011; Rukes et al., 2016b), showing an excellent match between the analytical results and numerical or experimental data, respectively. In the turbulent case, the LSA is not conducted on the base flow but on the time-averaged mean flow which is nonlinearly modified by turbulent stresses, entailing the global mode to stably oscillate at a limit cycle (Barkley, 2006). To accurately predict the PVC in turbulent environments, the direct influence of the turbulent stresses on the global mode needs to be accounted for with a turbulence model. It has been demonstrated that a Boussinesq's eddy viscosity model calibrated against the Reynolds stresses is suitable (Crouch et al., 2007, 2009; Meliga et al., 2012b; Mettot et al., 2014; Meliga et al., 2016; Rukes et al., 2016a; Tammisola \& Juniper, 2016). Recently, global LSA in conjunction with its adjoint counterpart has become more common. The combination of direct and adjoint analyses is particularly useful for localizing regions where the instability is sensitive to modifications and for revealing the 'wavemaker' of the instability which is responsible for its continuous self-excitation (Giannetti \& Luchini, 2007; Tammisola \& Juniper, 2016; Kaiser et al., 2017).

In contrast to round jets, stability analyses for annular swirling jets are less common. Instability-related studies focus mainly on two-phase flows where a liquid annular jet emerges in a gas environment (Vadivukkarasan 
\& Panchagnula, 2017; Matas et al., 2018) or on reacting flows in combustor geometries (Terhaar et al., 2015). To the authors' knowledge, no studies exist for single-phase non-reacting annular swirling flows. In this paper we aim to close this knowledge gap by analysing the global modes and associated coherent structures of an annular jet at moderate swirl level. Not only the rarely observed double helix vortex breakdown in turbulent flow is analysed in detail, but also the coexistence of two global modes is demonstrated. Although the simultaneous occurrence of a single and double helical structure in the flow field has been reported by many papers previously (Oberleithner et al., 2011; Tammisola \& Juniper, 2016), where the latter is a second harmonic of the former, this study shows that both structures are independent global modes and hence not harmonics of each other. The three-dimensional structures and dynamics are investigated experimentally using time-resolved tomographic particle image velocimetry (Tomo-PIV) measurements. The flow fields are subsequently analysed using (spectral) proper orthogonal decomposition. The physics involved are studied by reconstruction of the flow field from the identified coherent modes and by a global stability analysis of the mean flow field. The organisation of the paper is as follows. The experimental setup and data processing techniques are described in $\S 2$. The flow field analysis and the identification of coherent modes and their dynamics are done in $\S 3$. The results of the global stability analysis are presented in $\S 4$ and compared to the experimentally observed flow structures resulting from the (spectral) proper orthogonal decomposition of the experimental data. Finally, $\S 5$ summarises the main conclusions.

\section{Experimental setup and data processing}

\subsection{The swirling jet facility}

The investigation is carried out in a water facility. A schematic view of the experimental setup, including the tomographic PIV setup, is shown in figure 1. The swirling jet facility used in this study resembles a typical bluff body combustor geometry (figure 1a). The swirl generator is designed by the International Flame Research Foundation (IFRF) (Dugué \& Weber, 1992). The fluid is supplied by a pump and subsequently divided into six equal parts before entering the swirl generator through six evenly distributed radial inlets. The arrows in the figure indicate the flow direction. The flow passes a moveable block swirler which consists of twelve adjustable guide vanes that allow the amount of swirl to be changed. After flowing through an annular channel with an inner diameter $D_{i}=18 \mathrm{~mm}$ and an outer diameter $D_{o}=27 \mathrm{~mm}$, the jet issues in an octagonal water-filled tank which has a cross-sectional size of $600 \mathrm{~mm}\left(\approx 22 D_{o}\right)$ and a height of $800 \mathrm{~mm}\left(\approx 30 D_{o}\right)$. The height of the annular nozzle inside the octagonal bottom floor is $H=20 \mathrm{~mm}$. The tank is made of PMMA (acrylic glass) to ensure full optical access for illumination and tomographic imaging and the dimensions are large enough to resemble the conditions of a free jet. The symmetry axis of the jet is aligned with the y-axis in the measurement coordinate system with the origin located in the center and at the end of the inner tube. The velocity components in the $x, y$ and $z$ directions are labeled as $u, v$ and $w$, respectively. The working fluid is water with a density of 998.1 $\mathrm{kg} / \mathrm{m}^{3}$ and kinematic viscosity of $0.9915 \mathrm{~mm}^{2} / \mathrm{s}$. The flow rate is regulated by a frequency controlled submersible pump and the flow rate is fixed at $18 \mathrm{l} / \mathrm{min}$. This results in a Reynolds number of 8,500 based on the hydraulic diameter of the annular jet $\left(D_{h}=9 \mathrm{~mm}\right)$ and mean outlet axial velocity $v_{0}=0.94 \mathrm{~m} / \mathrm{s}$. The integrated flow rate based on the measured axial velocity profile at the nozzle outlet is within $3 \%$ of the direct measurement by the rotameter. In this study, the amount of swirl is characterised by the swirl number $S$, which is defined as the ratio of the flux of tangential and axial momentum times outer radius,

$$
S=\frac{\int_{0}^{2 \pi} \int_{\frac{D_{o}}{2}}^{\frac{D_{i}}{2}} \rho \overline{v v_{\theta}} r^{2} d r d \theta}{\frac{D_{o}}{2} \int_{0}^{2 \pi} \int_{\frac{D_{i}}{2}}^{\frac{D_{o}}{2}} \rho \bar{v}^{2} r d r d \theta},
$$

where the azimuthal velocity component $v_{\theta}$ is calculated based on averaging of the profiles at the nozzle exit in the azimuthal direction and the overbar denotes time-averaged quantities. In this study, the swirl number is kept constant at $S=0.36$.

\subsection{Data acquisition}

The flow field is measured using time-resolved Tomo-PIV. A schematic view of the measurement system is shown in figure 1b. Neutrally buoyant polyamide spherical particles of $56 \mu$ m mean diameter were employed as tracer particles at a concentration of around 0.65 particles per $\mathrm{mm}^{3}$. The flow was illuminated by a double-pulse Nd:YLF laser (Quantronix Darwin Duo, $2 \times 25 \mathrm{~mJ} /$ pulse at $1 \mathrm{kHz}$ ) with a wavelength of $527 \mathrm{~nm}$. The light scattered by the particles was recorded by a tomographic system composed of four HighSpeedStar 6 CMOS cameras (1024 $\times 1024$ pixels, 5400 frames/s, pixel pitch of $20 \mu \mathrm{m}$ ). Each camera was equipped with a Nikon $105 \mathrm{~mm}$ focal objective with a numerical aperture $\mathrm{f} \#=32$ to allow focused imaging of the illuminated particles. The cameras were linearly arranged in a horizontal plane with a viewing angle of $90^{\circ}$, where the two outer cameras were 


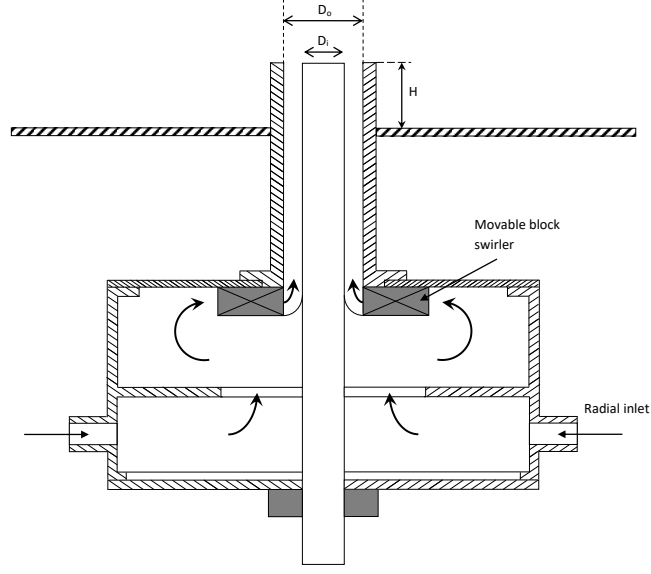

(a) Detail of the nozzle geometry and the swirl generator used.

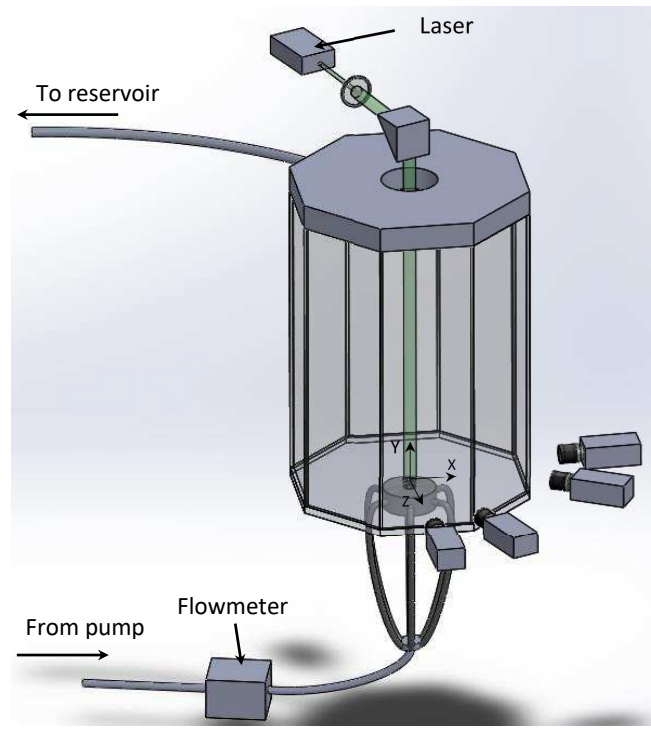

(b) General view of the tomographic PIV setup.

Figure 1: Schematic view of the experimental setup.

positioned/oriented along the $x$ and $z$ directions, respectively. The laser beam is transformed by the use of optics into a cylindrical volume with a diameter of $1.2 D_{o}$ and the height of the measurement domain is $1.8 D_{o}$, resulting in a digital resolution of 21.6 pixels $/ \mathrm{mm}$. The choice of a cylindrical measurement volume eliminated the need for a lens-tilt mechanism to comply with the Scheimpflug condition. Moreover, the use of a cylindrical volume yields a more favorable condition for the accurate volumetric reconstruction because the particle image density does not change with the viewing angle along the azimuth and decreases when moving toward the periphery of the jet. The average particle image density is approximately 0.045 particles per pixel (ppp). The images were captured in a single-frame mode at a recording frequency of $2.5 \mathrm{kHz}$ (which is about two orders of magnitude larger than the frequencies of the coherent flow structures studied) to enable the visualization of the time-series phenomena. A total of $N=5000$ samples are taken to obtain a sufficient amount of data for the decomposition analysis. This corresponds to a total acquisition time of 2 seconds, which is more than 50 times the precession period.

Image pre-processing, volume calibration, self-calibration, reconstruction and three-dimensional cross-correlationbased interrogation were performed in LaVision DaVis 8.1.6. A three-dimensional calibration target was scanned through the measurement volume in order to obtain the mapping function between the camera and the laboratory coordinate systems. The initial calibration was then refined by using the volume self calibration technique (Wieneke, 2008), yielding a misalignment of less than 0.05 pixels. The raw images were pre-processed with background intensity removal and particle intensity normalization. The reconstruction of the tomograms was performed by use of MLOS initialization (Atkinson \& Soria, 2009) and 10 CSMART iterations with Gaussian smoothing after each iteration. The particle images were then interrogated using windows of final size $48 \times$ $48 \times 48$ voxels with an overlap factor of $75 \%$, resulting in a vector spacing of $0.56 \mathrm{~mm}$ in each measurement direction. The dimensions of the measurement volume are 72 points in the $x$ and $z$ direction and 89 points in the $y$ direction. The measurement error of the instantaneous velocities is $0.04 v_{o}$ and the error on the time-averaged velocities is $0.005 v_{o}$. The reader is referred to Percin et al. (2017) for a more detailed discussion regarding the quality of the data and an error analysis.

\subsection{Large scale flow structure extraction}

Tomographic PIV measurements provide a vast amount of measurement data. The challenge to analyse these data is to separate turbulent or apparently stochastic features from other coherent motions in the flow field. Usually, these coherent motions have much longer time and much larger length scales compared to stochastic motions of fine-scale turbulence. As such, they can be overlooked in data analysis as they are often 'masked' by the latter ones. Several methods exist for the extraction of large-scale flow structures from turbulent flow fields. The most widely used ones are classical Fourier decomposition, proper orthogonal decomposition (POD) or its variants (Lumley, 1970; Sirovich, 1987; Boreé, 2003), dynamic mode decomposition (Rowley et al., 2009; Schmid, 2010; Williams et al., 2015) and very recently spectral proper orthogonal decomposition (SPOD) (Sieber et al., 2016). In this study, we apply snapshot POD and SPOD. In both techniques, the velocity field is decomposed into a set of spatial modes $\boldsymbol{\Phi}_{i}(\boldsymbol{x})$ and temporal coefficients $a_{i}(t)$ as 


$$
\boldsymbol{v}(\boldsymbol{x}, t)=\overline{\boldsymbol{v}}(\boldsymbol{x})+\boldsymbol{v}^{\dagger}(\boldsymbol{x})=\overline{\boldsymbol{v}}(\boldsymbol{x})+\sum_{i=1}^{N} a_{i}(t) \boldsymbol{\Phi}_{i}(\boldsymbol{x}),
$$

where $\boldsymbol{v}$ is a velocity vector with components $u, v$ and $w$. The overbar denotes time averaged quantities and hence only the fluctuating velocity field is decomposed. For the $N$ snapshots in time obtained by Tomo-PIV, the temporal coefficients $\boldsymbol{a}_{i}=\left[a_{i}\left(t_{1}\right), a_{i}\left(t_{2}\right), \ldots, a_{i}\left(t_{N}\right)\right]^{T}$ and mode energies $\lambda_{i}$ can be obtained by solving the eigenvalue problem

$$
\boldsymbol{R} \boldsymbol{a}_{i}=\lambda_{i} \boldsymbol{a}_{i} ; \quad \lambda_{1} \geq \lambda_{2} \geq \cdots \geq \lambda_{n} \geq 0,
$$

where the elements of the correlation matrix $\boldsymbol{R}$ are given by

$$
R_{i, j}=\frac{1}{N}\left\langle\boldsymbol{v}^{\dagger}\left(\boldsymbol{x}, t_{i}\right), \boldsymbol{v}^{\dagger}\left(\boldsymbol{x}, t_{j}\right)\right\rangle .
$$

As spatial inner product $\langle$,$\rangle , the L^{2}$ norm is usually taken. The spatial modes are obtained by the projection of the fluctuating velocity fields onto the temporal coefficients as

$$
\boldsymbol{\Phi}_{i}(\boldsymbol{x})=\frac{1}{N \lambda_{i}} \sum_{j=1}^{N} a_{i}\left(t_{j}\right) \boldsymbol{v}^{\dagger}\left(\boldsymbol{x}, t_{j}\right) .
$$

If periodic coherent structures exist in the flow field, the matrix $\boldsymbol{R}$ has a diagonal wave-like structure, also named diagonal similarity (Sieber et al., 2016). In SPOD, this similarity is augmented by filtering the correlation matrix $\boldsymbol{R}$ along the diagonals using a simple low-pass filter. This introduces the correlation matrix $\boldsymbol{S}$, defined as

$$
S_{i, j}=\sum_{k=-N_{f}}^{N_{f}} g_{k} R_{i+k, j+k},
$$

with $\boldsymbol{g}$ a vector of length $2 N_{f}+1$ with filter coefficients and $N_{f}$ the filter width. The correlation matrix $\boldsymbol{S}$ can then substitute $\boldsymbol{R}$ in equation 3 and the velocity field is decomposed using $\boldsymbol{S}$ instead of $\boldsymbol{R}$. A study by Sieber et al. (2016) on different testcases shows that optimal results are obtained if the filter size is one or two times the period of the periodic structure in the flow field.

An important aspect in the flow field analysis and decomposition using POD/SPOD is the identification of linked modes. For instance, the dynamical behavior of periodic coherent structures, such as the PVC, is described by a mode pair. A mode pair is defined as two modes in the decomposition which have the same spectral content and a constant phase difference of $\pm \pi / 2$. As such, reconstructing the flow field with a mode pair describes the precession of a coherent structure in the flow. A linked mode pair $a_{i}(t)$ and $a_{j}(t)$ can be identified by looking at the harmonic correlation of the eigenvectors of the DMD decomposition of the temporal coefficients. More detailed information on the identification of the pairs can be found in the work of Sieber et al. (2016).

\subsection{Triple velocity decomposition.}

As the large-scale coherent vortical structures found in swirling flows precess around the central axis of the jet, a simple Reynolds decomposition of the flow field into a mean and fluctuating part is not appropriate. This decomposition makes no distinction between the contributions of the precession and turbulence to the fluctuating part of the velocity. A more appropriate decomposition under this circumstance is the triple velocity decomposition as first proposed by Hussain \& Reynolds (1970). In this decomposition, the instantaneous velocity field at time instant $t_{i}$ is decomposed into three parts: a time-averaged (mean) flow field $\overline{\boldsymbol{v}}$, a quasi-periodic component induced by large-scale coherent structures, $\widetilde{\boldsymbol{v}}$, and a quasi-stochastic turbulent component $\boldsymbol{v}^{\prime}$ according to

$$
\boldsymbol{v}\left(\boldsymbol{x}, t_{i}\right)=\overline{\boldsymbol{v}}(\boldsymbol{x})+\widetilde{\boldsymbol{v}}\left(\boldsymbol{x}, t_{i}\right)+\boldsymbol{v}^{\prime}\left(\boldsymbol{x}, t_{i}\right) .
$$

The large-scale coherent component $\widetilde{\boldsymbol{v}}$ can be found by reconstruction of the velocity with the relevant mode pairs of the (S)POD decomposition as

$$
\widetilde{\boldsymbol{v}}(\boldsymbol{x}, t)=\sum_{k}\left(a_{i}^{k}(t) \boldsymbol{\Phi}_{i}^{k}(\boldsymbol{x})+a_{j}^{k}(t) \boldsymbol{\Phi}_{j}^{k}(\boldsymbol{x})\right),
$$

where a mode pair $k$ consists of (S)POD modes with indices $i$ and $j$. The second order statistical moments $\overline{\widetilde{v}}_{l} \widetilde{v}_{m}$ can be calculated (taken into account the orthogonality of the temporal coefficients, i.e. $\overline{a_{i} a_{j}}=\lambda_{i} \delta_{i j}$ ) as

$$
\widetilde{\widetilde{v}_{l} \widetilde{v}_{m}}=\sum_{k}\left(\lambda_{i}^{k} \Phi_{i l}^{k} \Phi_{i m}^{k}+\lambda_{j}^{k} \Phi_{j l}^{k} \Phi_{j m}^{k}\right),
$$

where $l$ and $m$ are components of the vector $\boldsymbol{\Phi}$. 


\subsection{Linear stability analysis}

Global LSA is employed to model the coherent velocity fluctuations, which are then compared to the coherent velocity fluctuations determined by the SPOD approach. The method is based on an eigenmode analysis of the linear operator determining the evolution of perturbations on the mean flow field. The governing equations are derived from the incompressible Navier-Stokes equations and the incompressible continuity equation. Employing the triple decomposition ansatz (see section 2.4), Eqn. 7 is substituted into both of these equations and both are time-averaged and phase-averaged. By subtracting the time-averaged set of equations from the phase-averaged set of equations, one arrives at the governing equations for the coherent velocity fluctuations (Reynolds \& Hussain, 1972):

$$
\begin{aligned}
\frac{\partial \widetilde{\boldsymbol{v}}}{\partial t}+\widetilde{\boldsymbol{v}} \cdot \nabla \overline{\boldsymbol{v}}+\overline{\boldsymbol{v}} \cdot \nabla \widetilde{\boldsymbol{v}}= & -\frac{\nabla \widetilde{p}}{\rho}+\nabla \cdot\left(\nu\left(\nabla+\nabla^{\top}\right) \widetilde{\boldsymbol{v}}\right) \\
& -\nabla \cdot(\boldsymbol{\tau}_{\mathrm{R}}+\underbrace{\boldsymbol{\tau}_{\mathrm{N}}}_{\approx 0}) \\
\nabla \cdot \widetilde{\boldsymbol{v}} & =0
\end{aligned}
$$

where $\boldsymbol{\tau}_{\mathrm{N}}=\widetilde{\boldsymbol{u}} \widetilde{\boldsymbol{u}}-\overline{\widetilde{\boldsymbol{u}} \widetilde{\boldsymbol{u}}}$ describes the nonlinear interactions of the perturbation with its higher harmonics (MantičLugo et al., 2015). This term has zero mean and can be interpreted as the fluctuation of the coherent Reynolds stresses due to nonlinear interaction with the higher harmonics. It is assumed that these interactions are weak and the nonlinear term is neglected in the following. Turton et al. (2015) states that the neglect of the nonlinear term is justified if the interaction of the fundamental with the higher harmonics is weak. In other words, it is assumed that the nonlinear energy transfer between the fundamentals and higher harmonics is weak compared to the energy transfer between mean and coherent field contained in the remaining linear terms of Eqn. 10 .

The other term $\boldsymbol{\tau}_{\mathrm{R}}=\left\langle\boldsymbol{v}^{\prime} \boldsymbol{v}^{\prime}\right\rangle-\overline{\boldsymbol{v}^{\prime} \boldsymbol{v}^{\prime}}=\widehat{\boldsymbol{v}^{\prime} \boldsymbol{v}^{\prime}}$ describes the fluctuation of the stochastic Reynolds stresses due to the passage of a coherent perturbation (Reynolds \& Hussain, 1972). This term must be modeled in order to close Eqn. 10.

As mentioned earlier, in the context of swirling flows with a PVC, it is now well established to use Boussinesq's eddy viscosity model as a closure (Rukes et al., 2016a; Oberleithner et al., 2015; Tammisola \& Juniper, 2016). This is done here as well. It is also common to assume that the coherent fluctuations of the turbulent kinetic energy are small enough to be negligible (Reynolds \& Hussain, 1972). With that assumption one arrives at:

$$
\boldsymbol{\tau}_{\mathrm{R}}=\widetilde{\boldsymbol{v}^{\prime} \boldsymbol{v}^{\prime}}=-\nu_{\mathrm{t}}\left(\nabla+\nabla^{\top}\right) \widetilde{\boldsymbol{v}}
$$

The unknown eddy viscosity is calculated from the measured velocity fields of the experiment. As the turbulence of the swirling jet is highly anisotropic, the approach in Eqn. 12 yields six independent eddy viscosities. A reasonable compromise among the six eddy viscosities can be achieved by using a least-square fit over all resolved stochastic Reynolds stresses (Ivanova et al., 2013):

$$
\nu_{\mathrm{t}}=\frac{\left\langle-\overline{\boldsymbol{v}^{\prime} \boldsymbol{v}^{\prime}}+2 / 3 \cdot k \boldsymbol{I}, \overline{\boldsymbol{S}}\right\rangle_{\mathrm{F}}}{2\langle\overline{\boldsymbol{S}}, \overline{\boldsymbol{S}}\rangle_{\mathrm{F}}}
$$

where $\langle\cdot, \cdot\rangle_{\mathrm{F}}$ is the Frobenius inner product, $k$ is the turbulent kinetic energy, $\boldsymbol{I}$ is the identity tensor and $\overline{\boldsymbol{S}}=1 / 2 \cdot\left(\nabla+\nabla^{\top}\right) \overline{\boldsymbol{v}}$ is the mean strain rate tensor. The eddy viscosity is then simply added to the kinematic viscosity to form an effective viscosity $\nu_{\mathrm{eff}}=\nu+\nu_{\mathrm{t}}$. The effective viscosity then replaces the kinematic viscosity in Eqn. 10.

The global LSA examines flows which are inhomogeneous in two or three spatial dimensions. These are typically named bi-global and tri-global LSA (Theofilis, 2011). For the present work, a bi-global analysis suffices due to the homogeneity of the mean flow along the azimuthal direction. Equations 10 and 11 are solved with a normal mode ansatz in cylindrical coordinates:

$$
\widetilde{\boldsymbol{q}}(\boldsymbol{x}, t)=\hat{\boldsymbol{q}}(\boldsymbol{x}, r) e^{\mathrm{i}(m \theta-\lambda t)}+\text { c.c. }
$$

where $\hat{\boldsymbol{q}}$ are the complex spatial amplitude functions of the velocities and the pressure, $m$ is the azimuthal wave number and $\lambda$ is the complex frequency. Discretisation and rearrangement leads to a linear generalized eigenvalue problem with $\lambda$ as eigenvalue. Solving the eigenvalue problem provides the eigenmodes $\hat{\boldsymbol{q}}$, each accompanied with one complex eigenvalue $\lambda$, respectively. The eigenvalue consists of a real part $(\lambda)=\omega$ that corresponds to the angular frequency of the mode and of an imaginary part $(\lambda)=\sigma$ corresponding to the temporal growth rate. In the eigenspectrum, an oscillator mode at the limit cycle, such as the PVC, is expected to be an eigenvalue isolated from any continuous eigenvalue branch and approximately marginally stable $(\sigma \approx 0)$ since the instability neither grows nor decays (Barkley, 2006). With that criterion and the known frequency from the experiment, the single helix and the double helix vortex breakdown mode can be identified. 


\begin{tabular}{cccc} 
Inlet & Outlet & Walls & Axis \\
\hline$\hat{v}=0$ & $\partial \hat{v} / \partial n=0$ & $\hat{v}=0$ & $\hat{v}=0$ \\
$\hat{v}_{r}=0$ & $\partial \hat{v}_{r} / \partial n=0$ & $\hat{v}_{r}=0$ & $\partial \hat{v}_{r} / \partial r=0$ \\
$\hat{v}_{\theta}=0$ & $\partial \hat{v}_{\theta} / \partial n=0$ & $\hat{v}_{\theta}=0$ & $\partial \hat{v}_{\theta} / \partial r=0$ \\
$\hat{p}=0$ & $\partial \hat{p} / \partial n=0$ & $\partial \hat{p} / \partial n=0$ & $\hat{p}=0$
\end{tabular}

Table 1: Boundary conditions of the global direct LSA

To obtain the adjoint modes, denoted with $(\cdot)^{+}$, the direct eigenvalue problem is reformulated as an adjoint eigenvalue problem being derived from the continuous formulation of the adjoint equations of Eqn. 10 and 11 (Luchini \& Bottaro, 2014). The adjoint mode can be interpreted as the receptivity of the direct mode with regard to a periodic forcing (Sipp et al., 2010). Furthermore, the structural sensitivity of an eigenvalue to mean flow modifications can be estimated via the Cauchy-Schwarz inequality from the direct and adjoint mode. The structural sensitivity is then defined by (Giannetti \& Luchini, 2007)

$$
\Lambda=\|\widetilde{\boldsymbol{v}}\| \cdot\left\|\widetilde{\boldsymbol{v}}^{+}\right\| .
$$

The regions of high structural sensitivity are interpreted as the wavemaker of the global instability. This is because the overlap of high amplitudes in both the direct and adjoint mode indicate the locations where the feedback among the coherent fluctuations is the strongest (Giannetti \& Luchini, 2007).

The Fortran code MAFIA (Paredes, 2014) is employed for the discretization of the linearized Navier-Stokes and continuity equations (Eqn. 10,11) and for solving the eigenvalue problem with the Arnoldi algorithm. A high-order finite difference scheme with nonuniformly distributed Chebyshev nodes is used for discretization. A total number of 8500 nodes is employed for a converged solution. The boundary conditions are set according to table 1. At the inlet, homogeneous Dirichlet boundary conditions for velocity and pressure are imposed since coherent fluctuations are not advected from upstream of the domain. At the outlet, homogeneous Neumann boundary conditions for the velocity and pressure are set since the domain is truncated at positions with remaining non-zero perturbations. For all walls, homogeneous Dirichlet boundary conditions are imposed for the velocity due to the no-slip and no-penetration condition. For the pressure, no physical boundary conditions exist. However, a compatibility condition from the governing momentum equations can be derived (Theofilis et al., 2004). Substituting the homogeneous Dirichlet conditions from the velocity into the linearized Navier-Stokes equation for the coherent perturbation, knowing that the eddy viscosity is zero per definition on the wall, taking the inner product with the unit normal vector $\boldsymbol{n}$ at the respective boundaries, and rearranging leads to:

$$
\frac{\partial p}{\partial n}=\rho \nu \frac{\partial^{2} v_{n}}{\partial n^{2}} .
$$

Assuming $\partial^{2} v_{n} / \partial n^{2} \approx 0$ provides homogeneous Neumann conditions for the pressure on the walls. For the adjoint LSA, the same boundary conditions are applied as for the direct modes except that the outlet conditions from table 1 are set to homogeneous Dirichlet conditions. In case of a converged adjoint solution, the adjoint eigenvalue $\lambda^{+}$of the PVC is the complex conjugate of the direct eigenvalue $\lambda$. By that, the adjoint eigenmode can be identified.

\section{Flow field analysis}

\subsection{Mean flow fields}

The time-averaged flow field is shown in figure 2, where radial profiles of $\bar{v} / v_{0}$ and $\bar{w} / v_{0}$ are depicted. The profiles of $\bar{v} / v_{0}$ are wake-like due to the presence of the CRZ since the centerbody acts as a bluff-body to the flow. This CRZ is toroidal due to the presence of the strong swirling component near the nozzle outlet as similarly observed by Vanierschot \& Van den Bulck (2008) and Sheen et al. (1996). Further downstream, a second recirculation zone appears, which is the vortex breakdown bubble. This recirculation zone is very weak as the maximum downward velocity is only $0.02 v_{0} \pm 0.005 v_{o}$. The azimuthal velocity profiles close to the central axis resemble a solid body rotation $(\bar{w}=\Omega y)$ for $x / D_{o} \approx 0.9$ with an angular rotational frequency of $\Omega=185 \mathrm{rad} / \mathrm{s}$ (figure $2 \mathrm{~b}$ ).

\subsection{Coherent structure extraction}

Recently, clear evidence of a double helical structure has been identified in the flow field of the same annular jet flow in both the velocity and pressure fields (Percin et al., 2017; Vanierschot et al., 2018). This double helix has the same features as the single helical PVC found in numerous other round or annular swirling jets: its branches are winded in the counterswirl direction and the helix itself precesses along the central axis in the 


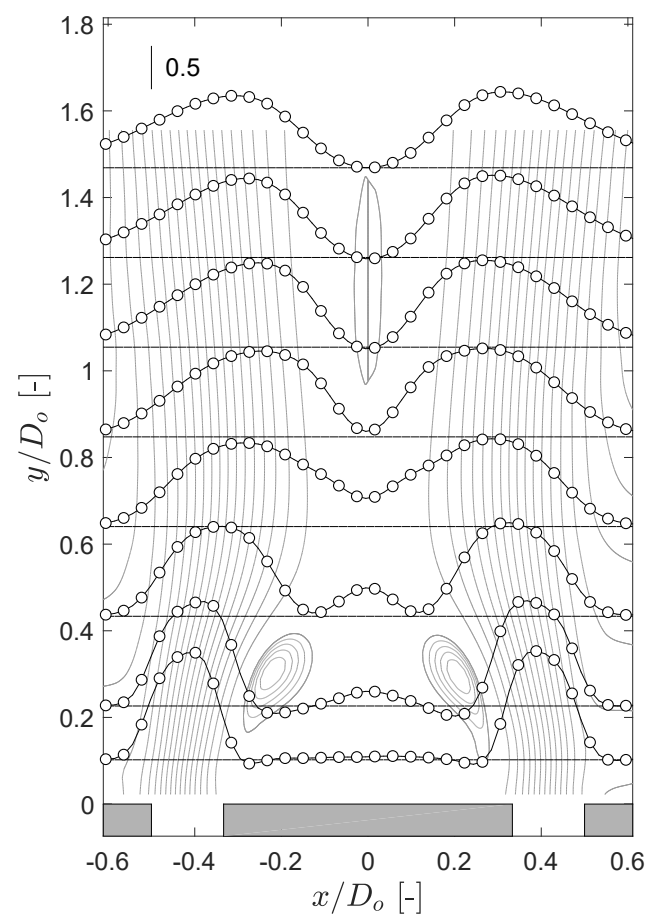

(a) Radial profiles of $\bar{v} / v_{0}$.

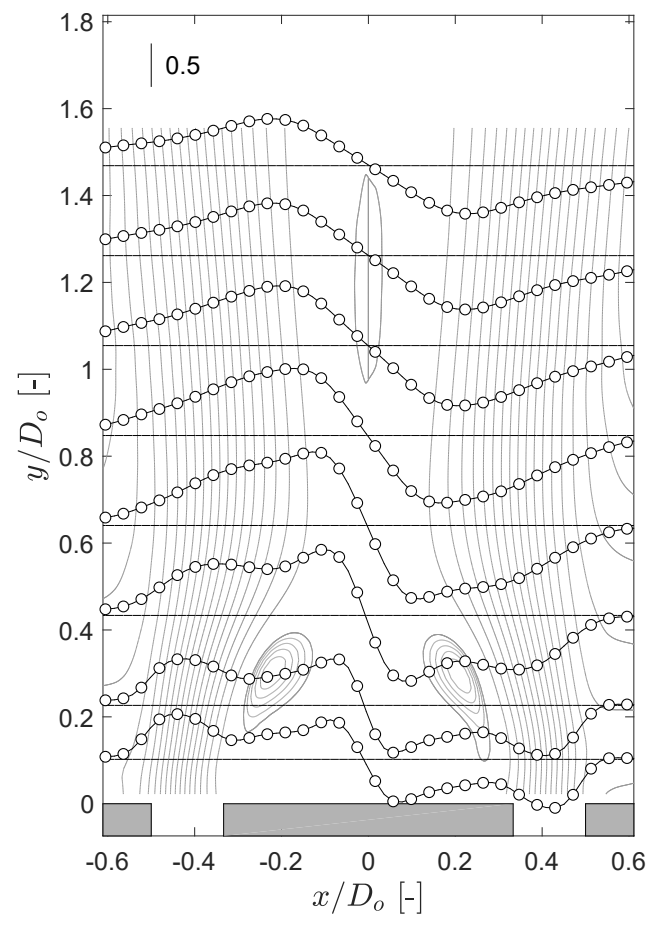

(b) Radial profiles of $\bar{w} / v_{0}$.

Figure 2: Time-averaged flow field. The grey lines in the background denote streamlines.

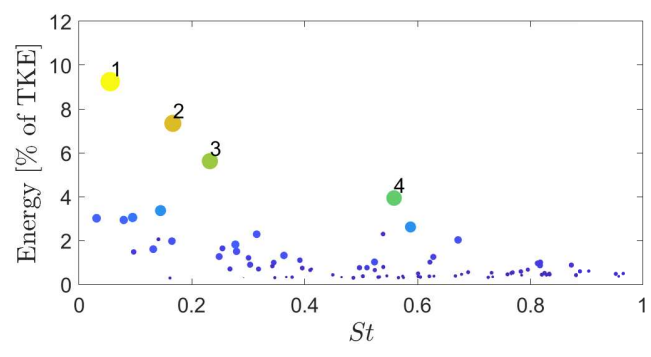

(a) Mode pair identification through POD.

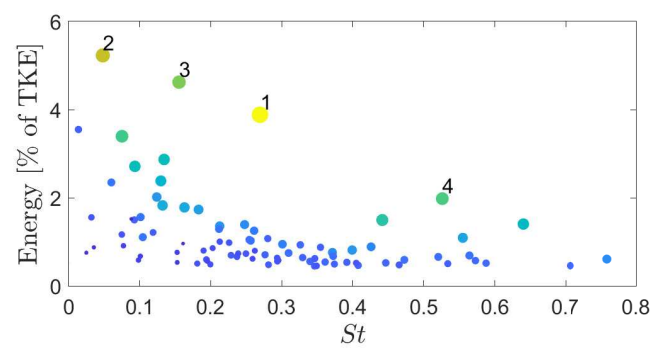

(b) Mode pair identification through SPOD.

Figure 3: Energy contribution of mode pairs to the dynamics of the flow using POD and SPOD decomposition. The diameter and color of the points are a measure for the harmonic correlation and the mode pairs are numbered with decreasing harmonic correlation strength. 


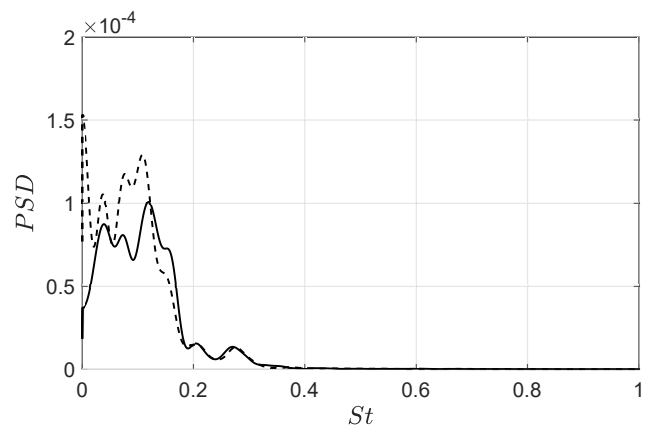

(a) Power Spectral Density of mode pair I (modes 1 and 2).

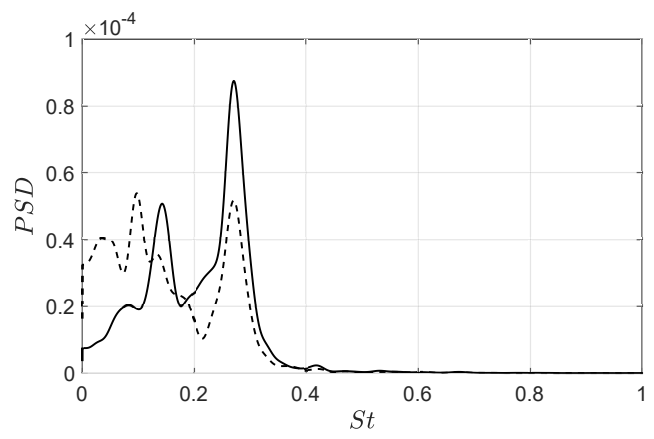

(c) Power Spectral Density of mode pair III (modes 5 and 6).

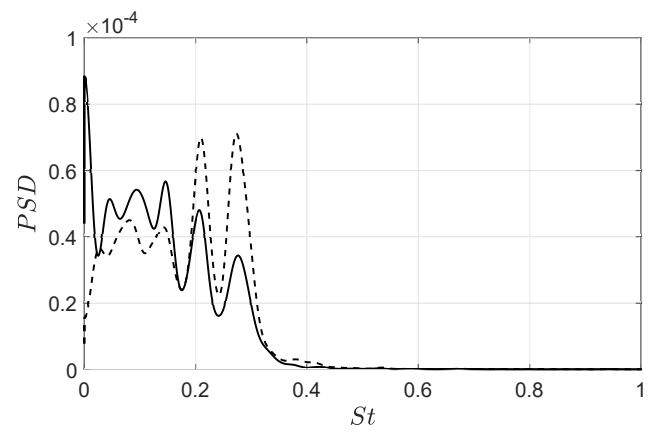

(b) Power Spectral Density of mode pair II (modes 3 and 4).

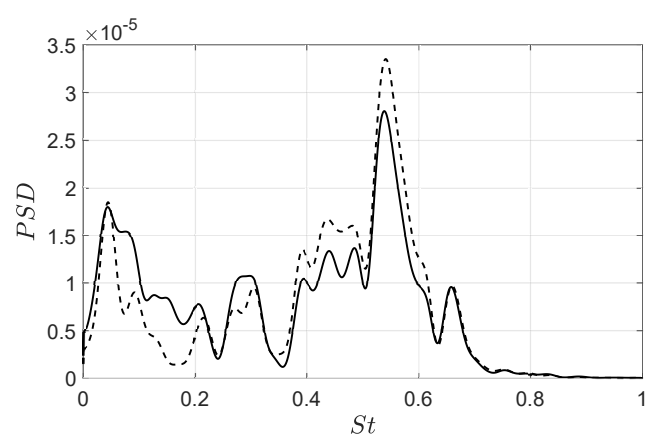

(d) Power Spectral Density of mode pair IV (modes 9 and 10.)

Figure 4: Power Spectral Density of the first four mode pairs in the POD decomposition. The solid line corresponds to the first mode of the pair and the dashed line to the second one.

swirl direction. Analysis of the velocity spectra at different locations in the flow field showed a dominant peak at $28.2 \mathrm{~Hz}$, corresponding to a Strouhal number based on the hydraulic diameter and mean jet outlet velocity, $S t=f D_{h} / v_{0}$ of 0.27 . POD analysis of the pressure field (Percin et al., 2017) allowed both a single and double helical structure to be identified, with corresponding Strouhal numbers of 0.28 and 0.56 . As the frequency of the double helix was double the one of the single helix, the authors speculated the double helix to be a higher harmonic of the single helix, as also shown by Oberleithner et al. (2011). Besides this peak, several other peaks have been observed, corresponding to motions of the wake behind the central body (Vanierschot \& Van den Bulck, 2008; Vanierschot et al., 2014). Hence, the annular jet is not only a very complicated flow field due to the presence of two interacting shear layers and its associated anisotropic structure of turbulence, but it also contains many coherent structures with different time and length scales. Moreover, the possible co-existence of single and double helical structures and their possible relation or interaction have not been resolved and require further analysis.

An analysis of the mode pairs in the POD and SPOD decomposition and their associated energy contribution is shown in figure 3. The four mode pairs with the highest harmonic correlation are labeled. Modes with a high harmonic correlation represent oscillating or precessing motions. In the POD decomposition in figure $3 \mathrm{a}$, these four modes also have the highest energy content and hence represent dominating large-scale structures in the flow field. However, considering the frequency spectra of the temporal coefficients of these four pairs (figure 4), not one single but several distinct peaks are present. For instance the peak found at $S t=0.27$ in the studies of Percin et al. (2017) and Vanierschot et al. (2018) can be found in modes pairs II and III. This indicates that contributions from several different structures in the flow with their own dynamics are present in a single mode pair. In other words, POD spreads out the coherent structures across multiple modes. Moreover, the double helical structure found in the flow by other studies can only be observed in mode pair IV (more specific mode 9 and 10 of the POD decomposition respectively). The energy content (around 3.9\%) is too low for the POD to separate it from other dynamical structures and noise in the data.

The mode pairs obtained by SPOD are shown in figure 3b. A filter width of 4 to 8 times the period of the motion to be studied was found to be the optimum. Increasing the filter width corresponds to narrowing the bandpass filter width in the spectrum of the coefficients (Sieber et al., 2016). If the filter size $N_{f}$ and hence the spectral attenuation width are too small, several other peaks can be found in the spectra (similar as for the conventional POD) and if the filter width is too large, the spectrum evolves towards a discrete Fourier transformation, which cannot take into account jitter in the frequency and modulation of the amplitude and is more prone to measurement noise. The optimal filter width obtained here is larger than the one found by Sieber 


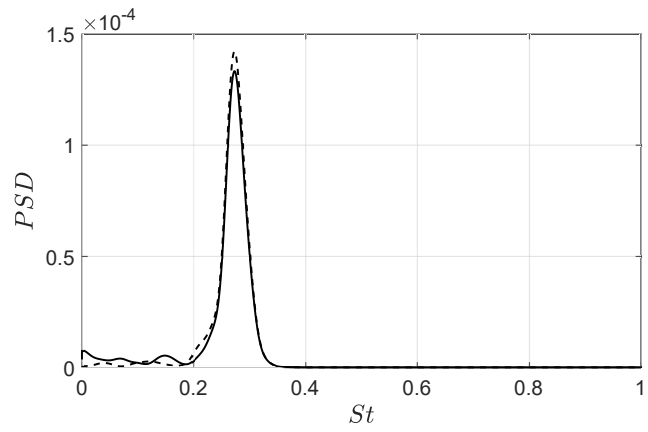

(a) Power Spectral Density of mode pair I (modes 6 and 7 ).

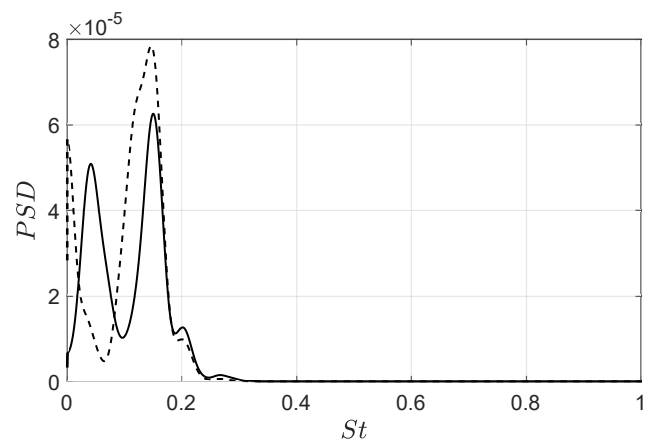

(c) Power Spectral Density of mode pair III (modes 3 and 4 ).

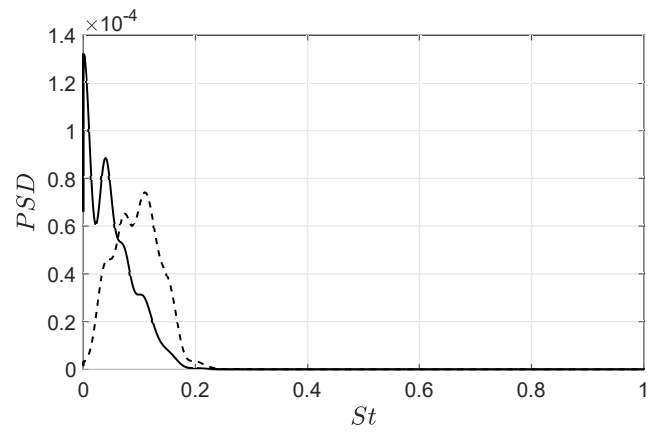

(b) Power Spectral Density of mode pair II (modes 1 and 2).

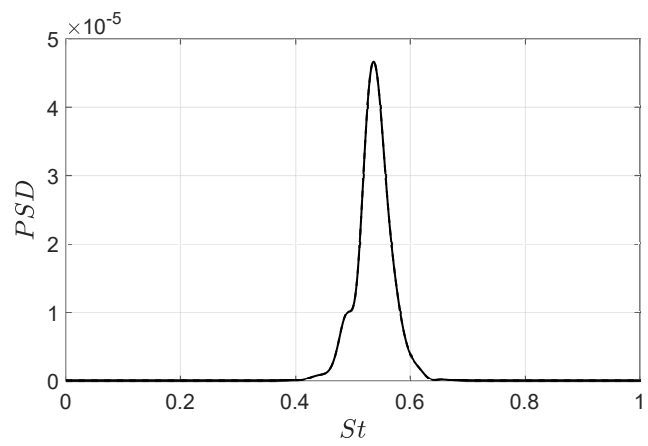

(d) Power Spectral Density of mode pair IV (modes 20 and 21).

Figure 5: Power Spectral Density of the first four mode pairs in the SPOD decomposition. The solid line corresponds to the first mode of the pair and the dashed line to the second one.

et al. (2016) indicating the strong dependence of $N_{f}$ on the flow topology. One could say that a good procedure would be to take the smallest value for which the frequency spectra of the time coefficients show no frequency content from other flow structures.

In figure $3 \mathrm{~b}$, the four pairs with the highest harmonic correlation are identical to POD. However, the individual order is different as is also the energy content. The PSD of the temporal coefficients of these pairs is shown in figure 5. Pairs II and III correspond to motions of the wake and bubble (Vanierschot \& Van den Bulck, 2008; Vanierschot et al., 2014). As the structures in these pairs have a rather low frequency content, the filter size is too small to enhance the diagonal similarity and several peaks are therefore still present in the spectrum. These structures were also found in the POD decomposition and will not be considered further to limit the scope of the study, which focuses on the helical instabilities in the jet. Besides those modes, two other mode pairs displaying distinct frequencies emerge: a dominant one at $S t=0.273 \pm 0.005$ with $3.9 \%$ energy content and a second one at $S t=0.536 \pm 0.005$ with $2 \%$ energy content. In the following, we go into more detail on the dynamics of mode pair I and IV.

\subsection{Mode pair I: a single helical PVC}

Mode pair I in the SPOD spectrum in figure $3 \mathrm{~b}$ consists of the 6 th and 7 th mode (according to energy ranking) in the SPOD decomposition. The temporal coefficients, scaled with the single mode energy, are shown in figure 6 a. From the figure it is clear that the phase shift between the two time coefficients is $\pi / 2$ as the minima and maxima of one coefficient corresponds to the zeros of the other one and vice versa. Also the circular shape of the Lissajous curves (phase portraits) in figure $6 \mathrm{~b}$ confirms this. Frequency spectra of the temporal coefficients (Figure 5a) show a clear peak at $28.5 \mathrm{~Hz}$ or $\mathrm{St}=0.273$ with equal magnitude for both coefficients of the pair. Figure 6 a reveals that the single helix is not always present in the flow. The amplitude evolves from zero towards the limit cycle and back in a seemingly random way. This evolution in amplitude suggests that the mode is a marginally stable one and it gets excited by turbulent fluctuations or due to stochastic changes of the mean flow field (slow drift modes). More general, in dynamical system theory the observation of a stable mode due to random forcing is also referred to as coherence resonance. Since the single helical structure is not always present in the flow, it has a relatively low time-averaged energy content, making it very hard to be detected by POD. The spatial structure of the mode pair is shown in figure $6 \mathrm{c}$ and is visualised using the Q-criterion (Jeong \& Hussain, 1995). Each mode consists of a double helical structure and the phase angle between the two modes is $\pi / 2$. Recombining the investigated mode pair with the mean flow field (figure $6 \mathrm{~d}$ ) shows a single helical structure 


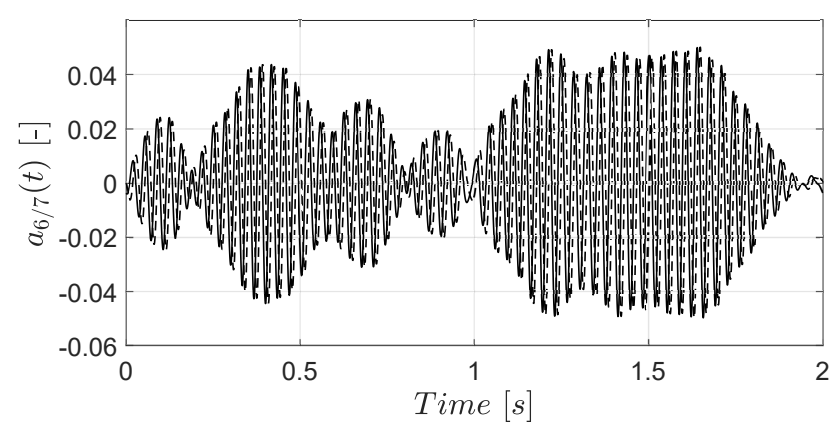

(a) Evolution of the temporal coefficients. Solid line: $a_{6}(t)$, dashed line: $a_{7}(t)$.

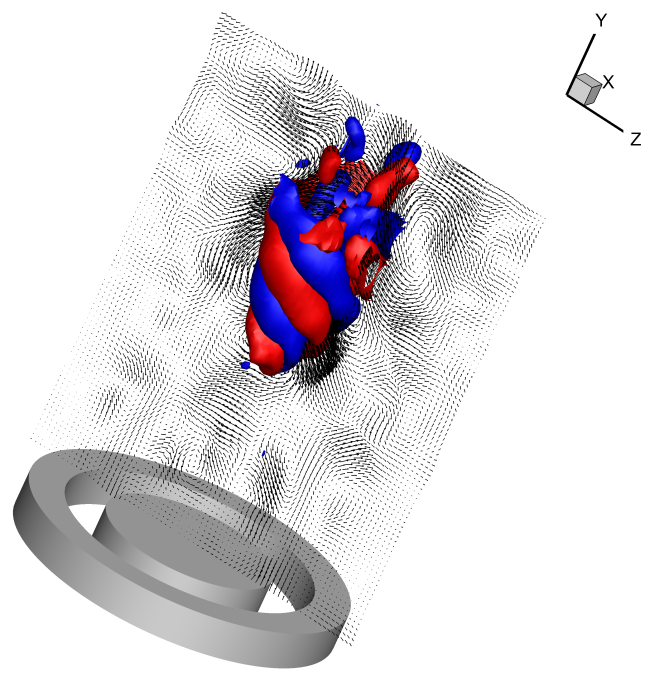

(c) Spatial structure of mode 6 and 7. Isocontours of $Q=0.251 / s^{2}$. Blue: mode 6 , Red: mode 7 .

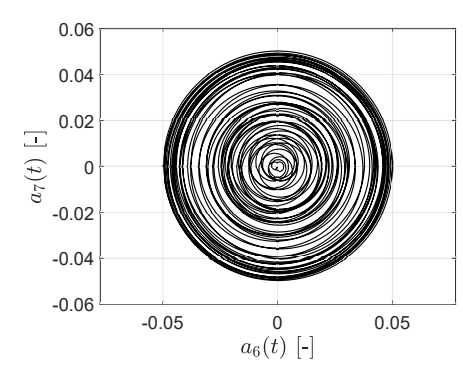

(b) Phase portrait.

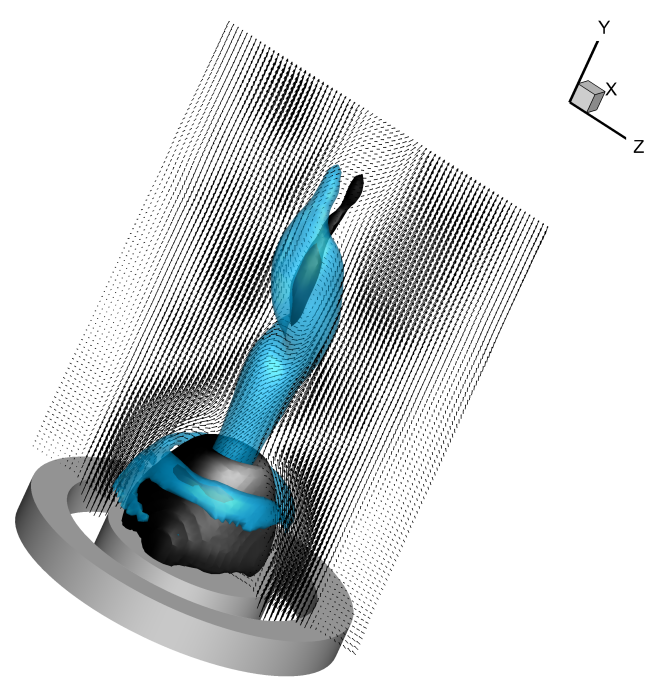

(d) Reconstructed velocity field with only mode pair one. The light blue lines are isovalues of $\mathrm{Q}=$ $0.041 / s^{2}$ and the black surfaces are iso-surfaces of zero axial velocity.

Figure 6: Temporal and spatial characteristics of mode pair I (mode 6 and 7 in the SPOD decomposition).

which is wrapped up in the counterswirl direction upstream of the vortex breakdown bubble (visualised by the downstream black iso-contour surface of zero axial velocity in the figure).

\subsection{Mode pair IV: a double helical PVC}

Mode pair IV in the SPOD spectrum in figure 3b consists of the 20th and 21st mode in the SPOD decomposition. The temporal coefficients are shown in figure 7a. Similar to mode pair I, the phase shift between the time coefficients is $\pi / 2$, again confirmed by the circles in the Lissajous curves (phase portraits) in figure $7 \mathrm{~b}$. Frequency spectra of the coefficients in figure $5 \mathrm{~d}$ show a clear peak at $56 \mathrm{~Hz}$ or $S t=0.536$ which is equal in magnitude for both coefficients. This frequency is double the frequency of mode pair I within measurement accuracy. Together with the observation that the azimuthal wavenumber is also double, one might think that the double helix is the first harmonic of the single helix. However figure 8 shows that mode pair IV is not a higher harmonic of mode pair I. If this would be the case, the Lissajous figure in figure 8a should have the shape of an eight. Nevertheless, plotting the phase portrait might be corrupted by intermittence and phase distortion of both modes and therefore a more detailed statistical analysis is given in figures $8 \mathrm{~b}$ and $\mathrm{c}$. If the two modes would be harmonics of each other, the plot of $\phi_{I V}$ versus $\phi_{I}$ should give the arrangement of the dots in 3 major diagonals, where $\phi_{I}=\arctan \left(a_{6} / a_{7}\right)$ and $\phi_{I V}=\arctan \left(a_{20} / a_{21}\right)$ are the phase angles of the mode pairs. The scattering of the data shows that this is clearly not the case. Also, if one would plot the statistical histogram of the variable $2 \phi_{I}-\phi_{I V}$, and the modes happen to be harmonics of each other, the fixed phase relation should give one clear peak in the histogram. Figure $8 \mathrm{c}$ shows that there is no statistical correlation and hence it can be concluded that both modes are not harmonics of each other.

Figure 7a shows that mode pair IV, is not always present in the flow, similar to the observations made for mode pair I. Comparison of figures $6 \mathrm{a}$ and $7 \mathrm{a}$ shows that there is no clear correlation between the appearance of the single and double helix in the flow field as both modes can coexist for some time intervals, while for other intervals only one of the two is present. Also, mode pair IV is more dynamic compared to mode pair I as the 


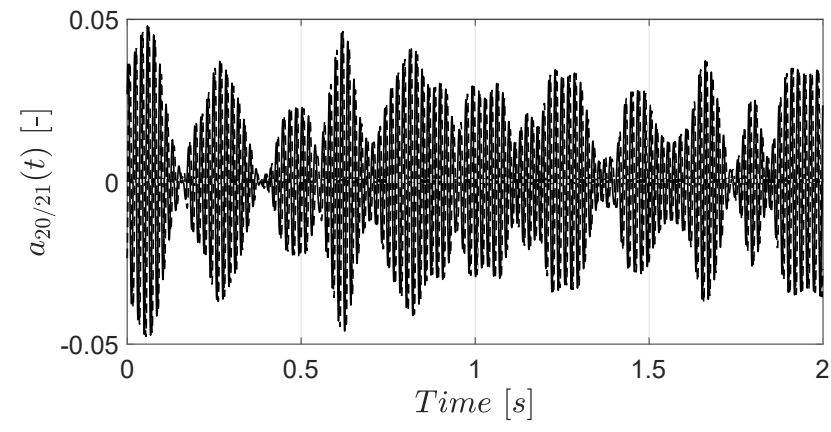

(a) Evolution of the temporal coefficients. Solid line: $a_{20}(t)$, dashed line: $a_{21}(t)$.

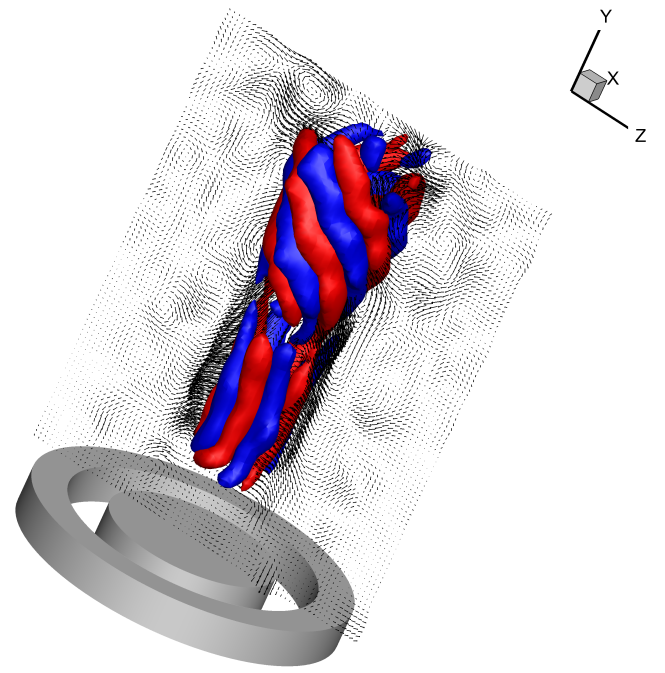

(c) Spatial structure of mode 20 and 21. Isocontours of $Q=0.51 / s^{2}$. Blue: mode 20, Red: mode 21.

Figure 7: Temporal and spatial characteristics of mode pair IV (mode 20 and 21 in the SPOD decomposition).

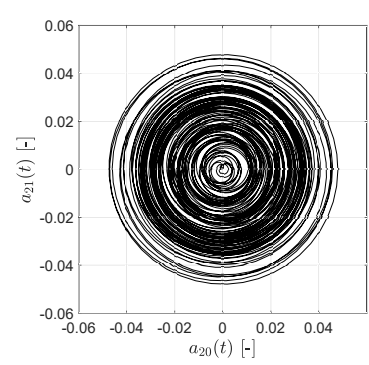

(b) Phase portrait.

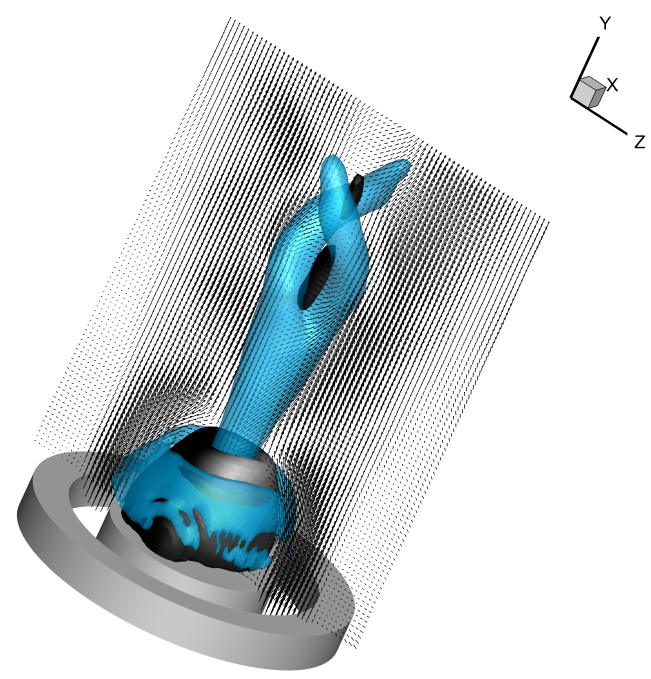

(d) Reconstructed velocity field with only mode pair IV. The light blue lines are isovalues of $\mathrm{Q}=$ $0.0351 / s^{2}$ and the black surfaces are iso-surfaces of zero axial velocity. 


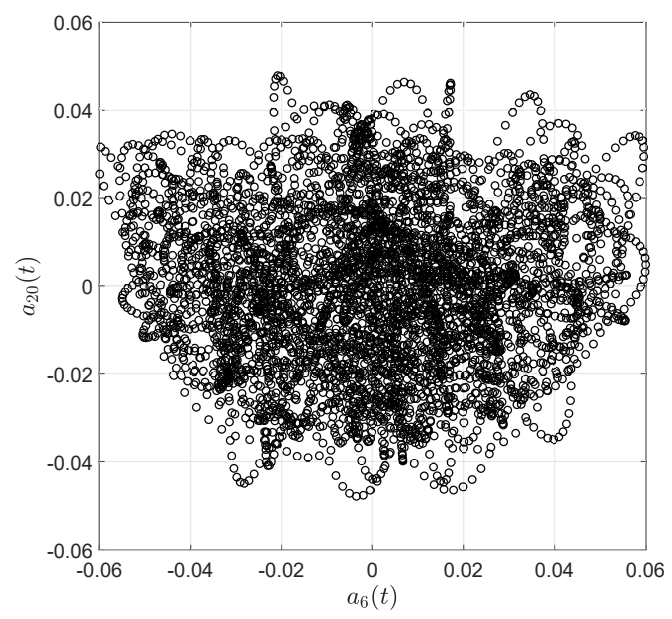

(a) First temporal coefficient of mode pair IV versus mode pair I.

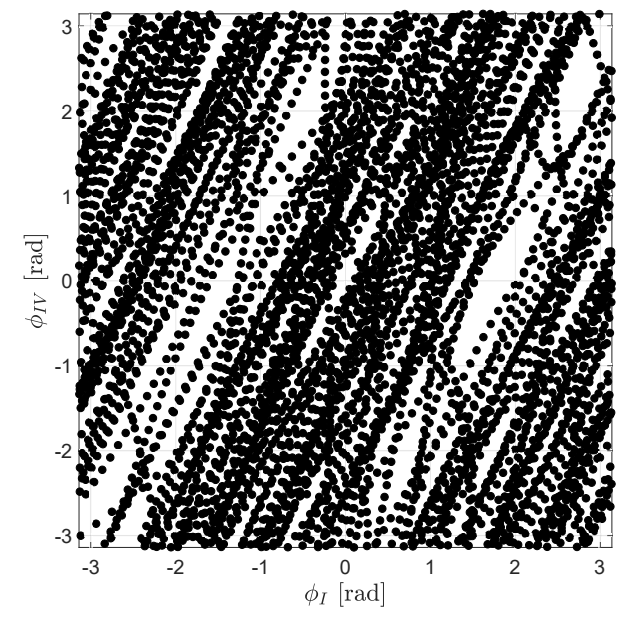

(b) Phase angle of mode pair I versus the one of mode pair IV.

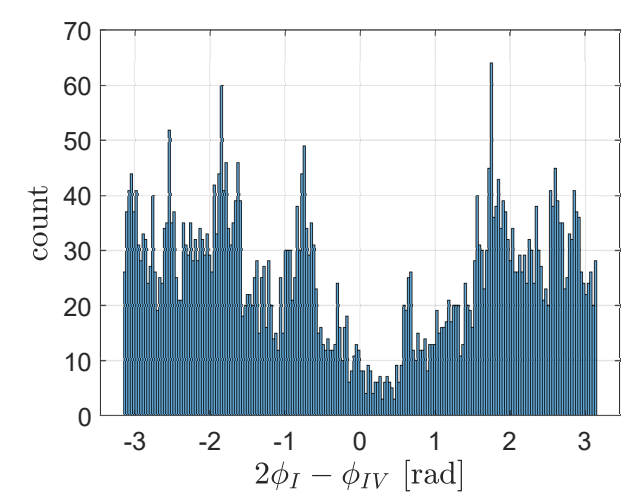

(c) Histogram of the phase difference of mode pair I and mode pair IV.

Figure 8: Analysis of the correlation between the phase angle of mode pair I versus the one of mode pair IV.

amplitude grows/decays more often. The spatial structure of mode pair IV is shown in figure 7c. It indicates two different regions where the vortical structures are oriented differently. In the breakdown region the mode has a helical structure, similar to mode pair I, but in the wake region, the vortical structures are oriented along the streamwise direction. The orientation of the vortices indicates different orientations of the mean strain rates that produce the vorticity. In the breakdown region, the superposition of axial and azimuthal velocities gives rise to helical vortical structures. In contrast, there is little axial velocity in the wake region behind the bluff body of the jet, which leads to a streamwise orientation of the vorticity. The mode shape indicates a linked dynamic in the two regions, which is in contrast to the single helical structure. Recombining the flow field (figure $7 \mathrm{~d}$ ) reveals a double-helical structure which is wrapped up in the counter-swirl direction upstream of the vortex breakdown bubble (visualised by the downstream black iso-contour surface of zero axial velocity in the figure). Once reconstructed in combination with the mean flow field, both structures precess at an angular frequency $\omega=2 \pi f / m$, where $m$ is the azimuthal wavenumber and $f$ is the frequency corresponding to the peak in the PSD of the corresponding temporal mode coefficients. This results in a phase rotation rate of $\omega=180 \mathrm{rad} / \mathrm{s}$ for both structures as the frequency peak of the double helix is more or less double the one of the single helix and the azimuthal wave number is also double. This value is very close to the bulk rotation rate of the jet at the axial location where the cores break up, $\omega=185 \mathrm{rad} / \mathrm{s}$, defined as the slope at the axis of the radial profiles of rotational velocity just upstream of the vortex breakdown bubble (figure $2 \mathrm{~b}$ ).

The dynamics of the two large-scale structures in the flow introduce extra mixing in the flow field. To quantify this mixing, figure 9 shows the contribution of both precessions to the second order statistics (called the coherent stresses) of the flow. The single helix has the highest coherent stresses near the region where the central vortex core breaks up in a single helix. Slightly upstream of the bubble, the horizontal velocity components $(u$ and $w)$ have the highest fluctuations, while for the axial velocity $v$, the fluctuations are highest in the shear layer between the vortex breakdown bubble and the jet. The double helix shows a different behavior. Both $u$ and $w$ show large values in a region immediate downstream of the CRZ and in the shear layer between the vortex breakdown bubble and the jet. Comparison with the statistical data of Percin et al. (2017) shows that despite the low energy content of the two precession motions (around 4 and $2 \%$ respectively), the impact on the second 


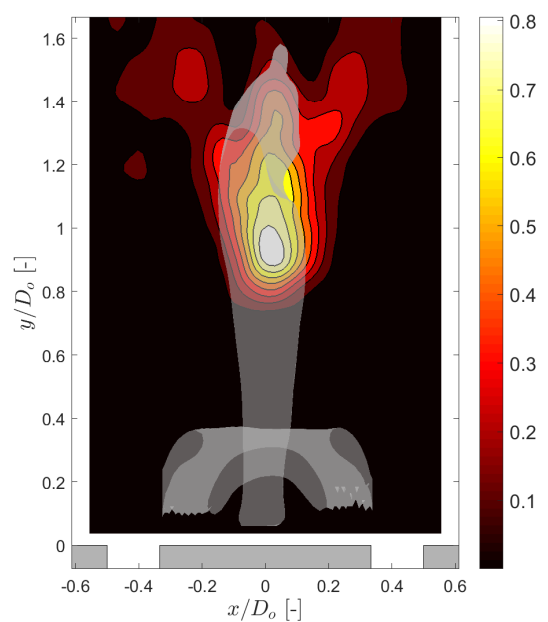

(a) $\overline{\widetilde{u} \widetilde{u}} / v_{0}^{2} \times 100$.

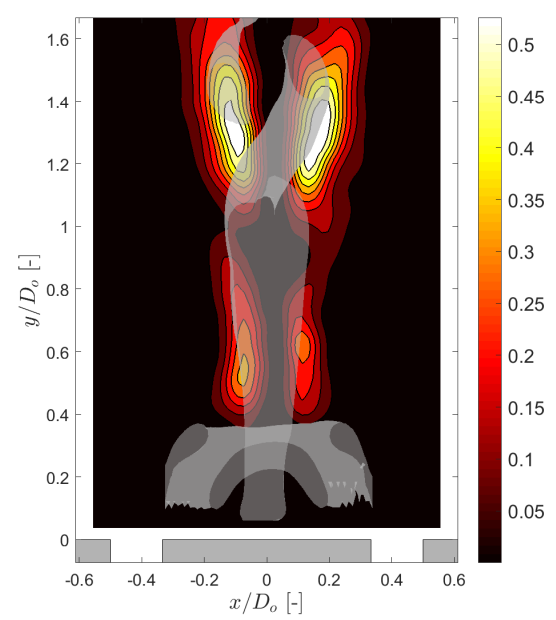

(d) $\overline{\widetilde{u}} \widetilde{u} / v_{0}^{2} \times 100$.

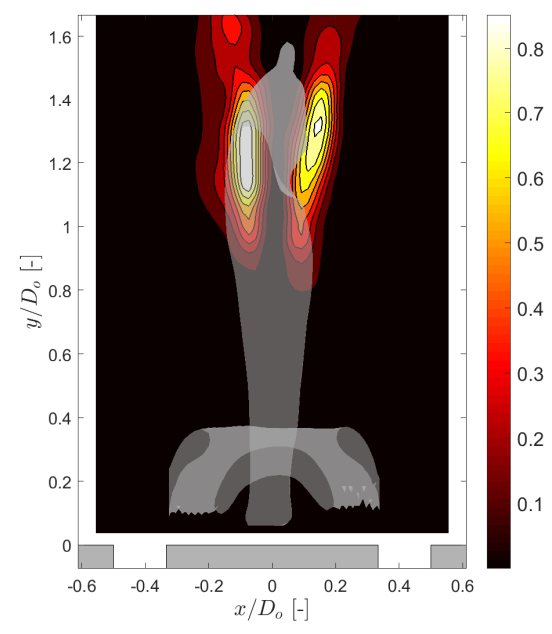

(b) $\overline{\widetilde{v} \widetilde{v}} / v_{0}^{2} \times 100$.

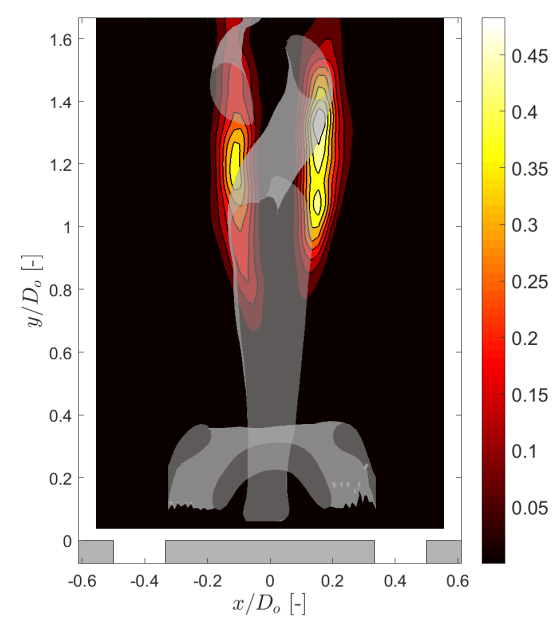

(e) $\overline{\widetilde{v} \widetilde{v}} / v_{0}^{2} \times 100$.

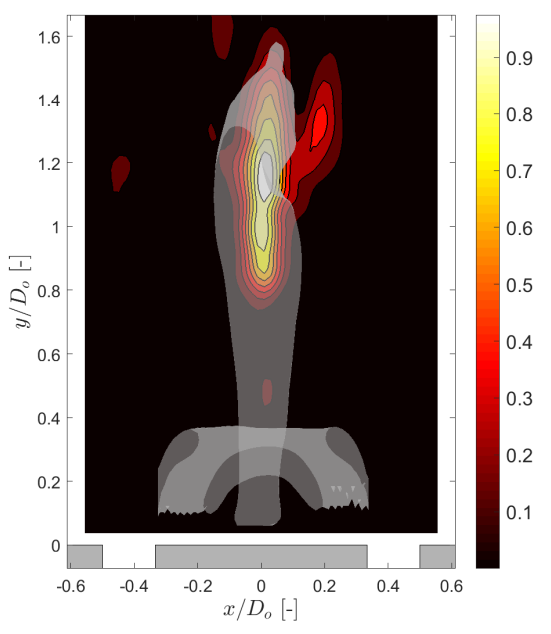

(c) $\overline{\widetilde{w} \widetilde{w}} / v_{0}^{2} \times 100$.

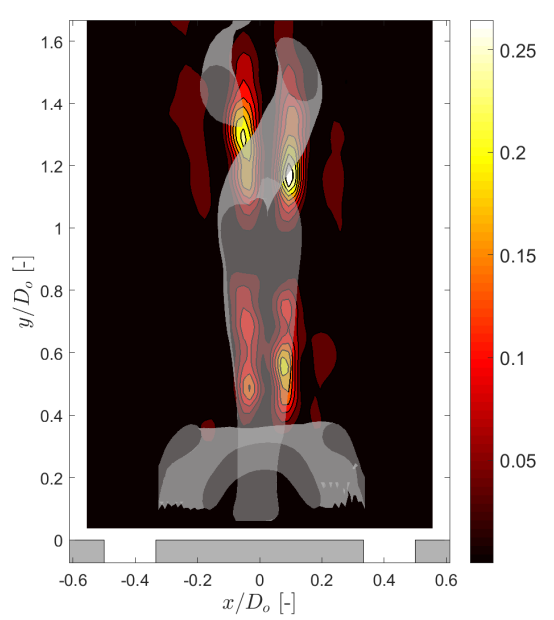

(f) $\overline{\widetilde{w} \widetilde{w}} / v_{0}^{2} \times 100$.

Figure 9: Coherent contribution to the second order statistics of the single (first row) and double helical (second row) precession.

order statistics in the near bubble region is significant with contributions of around $16 \%$ for $\overline{\widetilde{u}} \bar{u}$ and $\overline{\widetilde{v}}$ and of $30 \%$ for $\widetilde{w} \widetilde{w}$. Analog to the production of turbulent kinetic energy caused by mean shear, the contribution of the coherent flow component to the production of the kinetic energy can be written as

$$
P=-\overline{\tilde{v}}_{j} \tilde{v}_{i} \frac{\partial \bar{v}_{i}}{\partial x_{j}},
$$

where the expression is written in Einstein notation. The production of coherent kinetic energy for the two helical modes is shown in figure 10. High values indicate regions where the coherent structures extract energy from the mean flow. It can be seen that both the single and double helix extract energy from the shear layer between the vortex breakdown bubble and the jet, albeit somewhat more downstream for the double helix, while the former extracts more energy. The double helix also extracts a smaller amount of energy from the mean flow at the end of the CRZ.

\section{Results of the global linear stability analysis}

\subsection{Frequency, growth rate and mode structure}

The eigenvalues obtained by the global direct LSA are displayed in figure 11. The spectrum is entirely stable since the growth rate of every eigenvalue is below the stability limit with $\sigma<0$. The eigenvalues marked with filled symbols correspond to the selected modes of interest that represent the single- and double-helical mode in the best possible way within the constraints of the normal mode basis. Further elaborations on convergence and accuracy of the eigenspectrum are provided in Appendix A and B below. Considering the single-helical spectrum (azimuthal wavenumber $m=1$ ), the least stable eigenvalue represents the single-helical mode found in the 


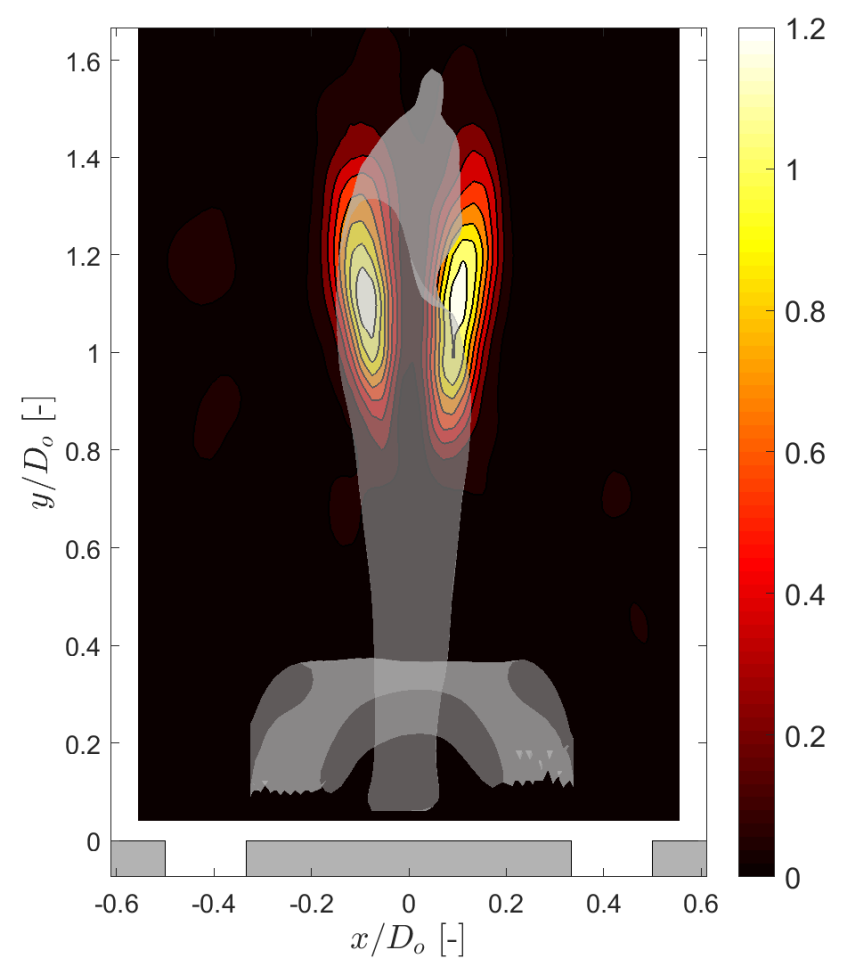

(a) Kinetic energy production $\mathrm{P}\left(\mathrm{m}^{2} / \mathrm{s}^{3}\right)$ of the single helix.

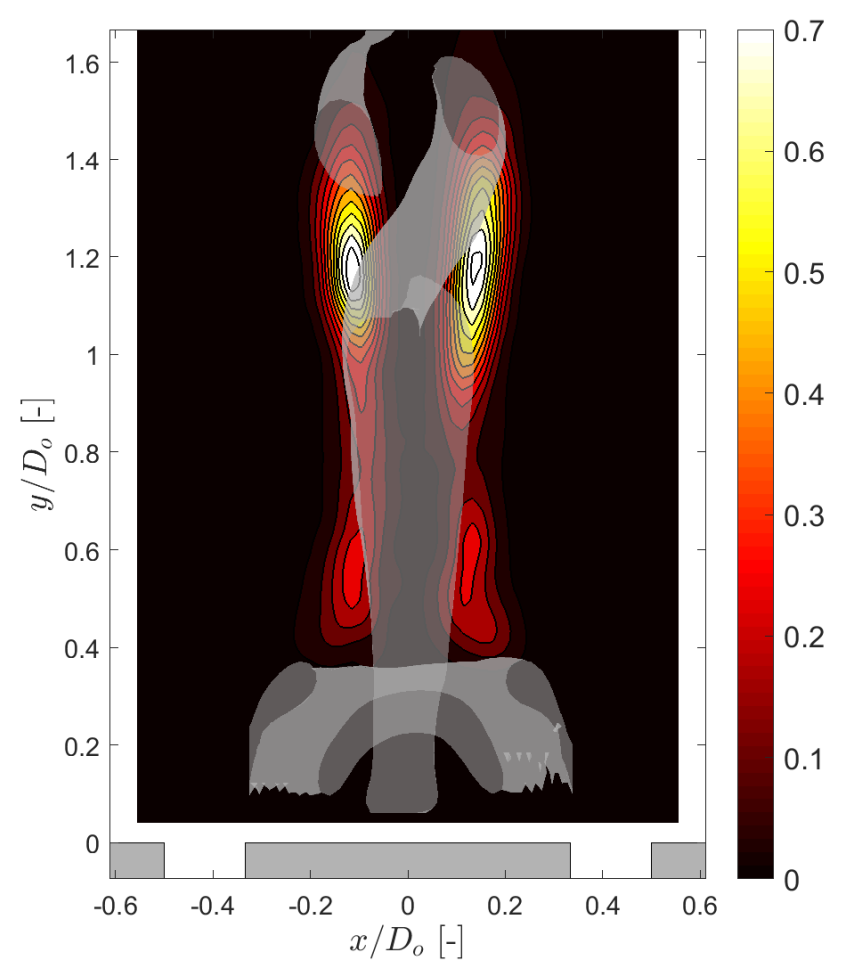

(b) Kinetic energy production $\mathrm{P}\left(\mathrm{m}^{2} / \mathrm{s}^{3}\right)$ of the double helix.

Figure 10: Kinetic energy production of the single and the double helix.

experiment. Its frequency of $S t=0.268$ is very similar to the experimentally measured frequency of $S t=0.273$ with a relative error of less than $2 \%$. For the double-helical spectrum (azimuthal wavenumber $m=2$ ), the eigenvalue with a frequency of $S t=0.464$ represents the double-helical mode found in the experiment. In this case, the relative error to the experimentally obtained Strouhal number is approximately 13\%. The larger mismatch can be attributed to the position of the wavemaker, related to regions of recirculation in conjunction with the strong mean flow gradients occurring in its vicinity. Since the predicted LSA frequency is quite sensitive with regard to changes of the swirl velocity in the wavemaker region, any measurement error of the Tomo-PIV has a significant impact. This is likely to be the cause for the frequency mismatch. This is further shown and discussed in Appendix A.

The growth rates being close but below the stability limit $(\sigma 0)$ reflect the property of both global modes existing only due to continuous stochastic forcing. The small negative values of the growth rates indicate that the modes only decay very slowly over large periods of time (note that the time scale of decay is more than an order of magnitude larger than the time scale of oscillation). However, for short periods of time, the modes can still transiently grow when stochastic forcing is applied. For the single-helical mode $(m=1)$, the growth rate is associated with an error due to domain truncation at the outlet boundary. A shift of the outlet boundary in the upstream direction up to $30 \%$ results in an increase of the negative growth rate (see Appendix C). This is associated with the truncation of the region of high structural sensitivity (shown and discussed further below), meaning that a significant part of the wavemaker centered around $y / D_{o} \approx 1.3$ is excluded. Therefore, it can be expected that the correct growth rate of the single-helical mode is actually even closer to the stability limit. In case of the double-helical mode $(m=2)$, no sensitivity of the growth rate due to domain truncation is identified. This is attributed to the primary wavemaker being located far upstream at the end of the recirculation bubble, concentrated around $y / D_{o} \approx 0.4$.

The spatial structure of both single- and double-helical modes, based on the $Q$ criterion, is shown in figure 12 . Comparing the mode of $m=1$ extracted via SPOD (see figure 6 ) to the mode predicted by global LSA, it is evident that a good match exists in some parts, while in other parts a slight mismatch can be observed. The inclination angle of the isosurfaces representing the counter-winding vortices is associated with the axial wavelength and it can be seen that prediction and measurement match very well. The increasing radial spread in the downstream direction is related to the convective growth and the LSA correctly captures this property. Additionally, the location where the mode starts to initially grow is predicted accurately as well. For $m=2$ (compared to figure 7), there are also clear similarities, although there is some mismatch too. The initiation of the double-helical mode far upstream at the end of the recirculation bubble is correctly predicted including the large axial wavelengths, resulting in a very small inclination angle of the counter-winding vortices. Downstream of the bubble, the mode decays at first, before it is again amplified in the axial direction. This trend is reproduced by the LSA mode as 


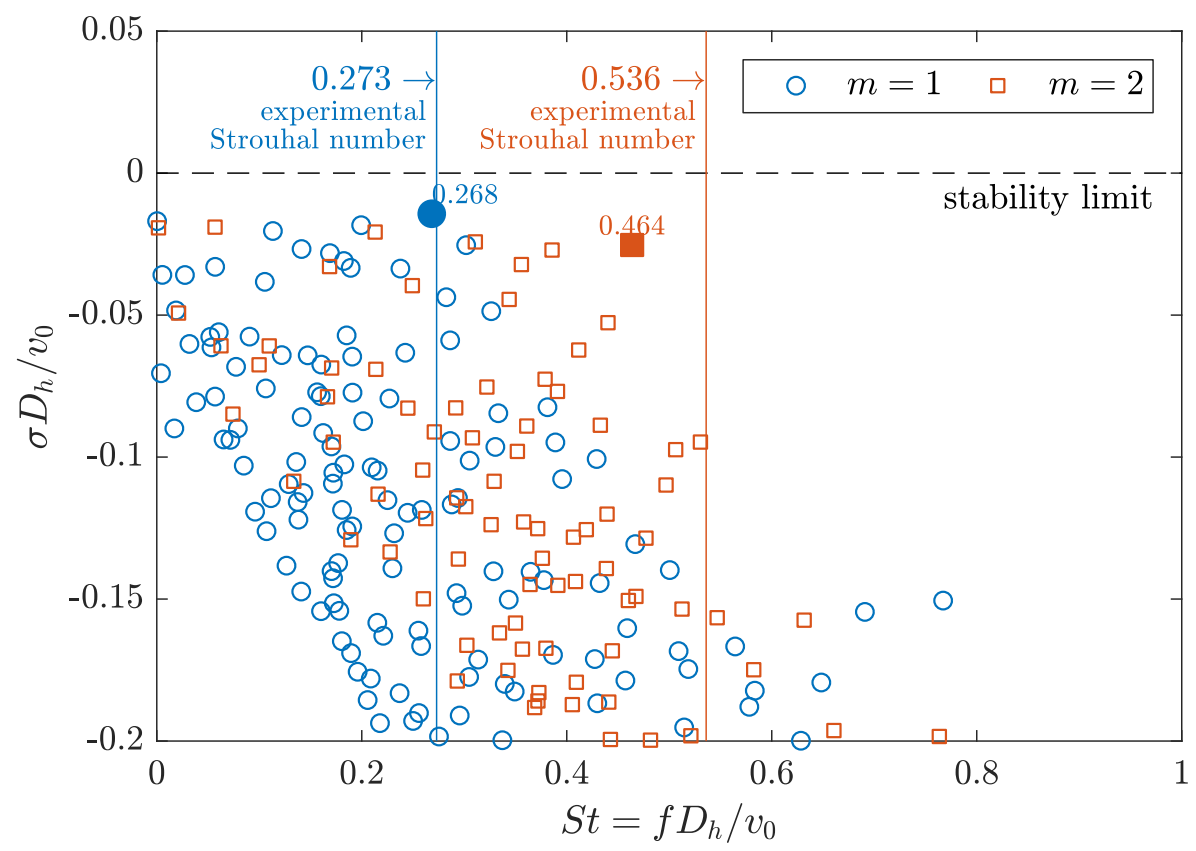

Figure 11: Eigenvalue spectrum with nondimensional growth rate $\sigma D_{h} / v_{0}$ over Strouhal number $S t$ obtained by global direct LSA of the mean flow for azimuthal wavenumber $m=1$ and 2 , filled markers $(\bullet$,$) denote the$ identified single-helical and double-helical mode, vertical solid lines denote the experimentally measured Strouhal numbers and the horizontal dashed line denotes the stability limit.

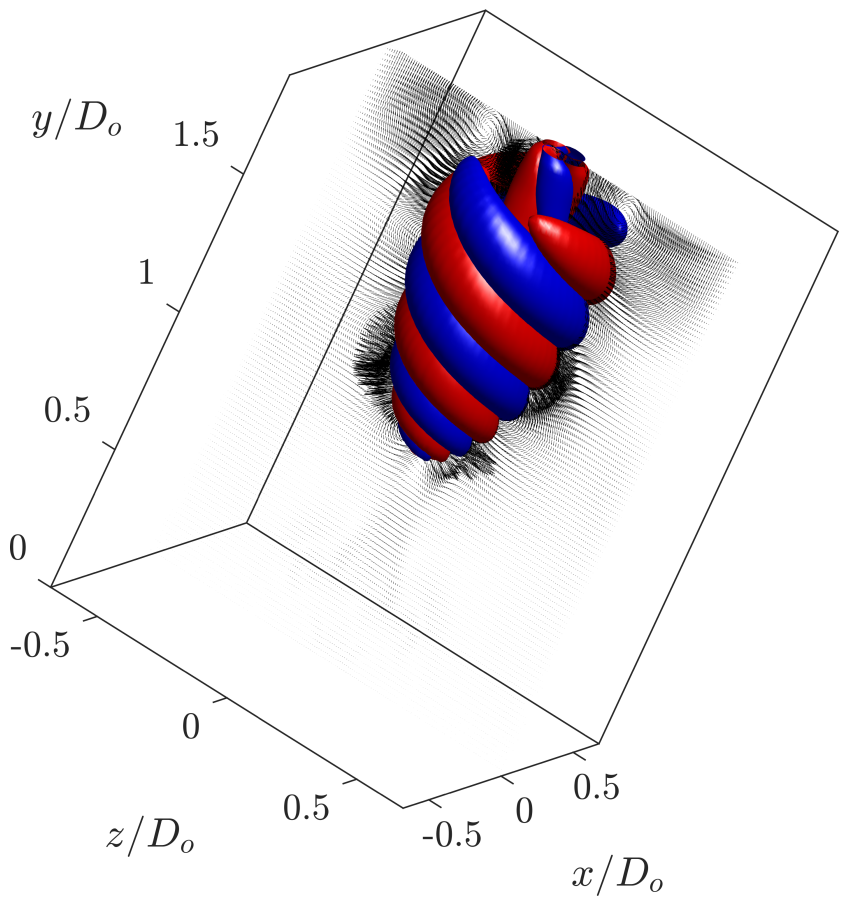

(a) Single-helical mode $(m=1)$

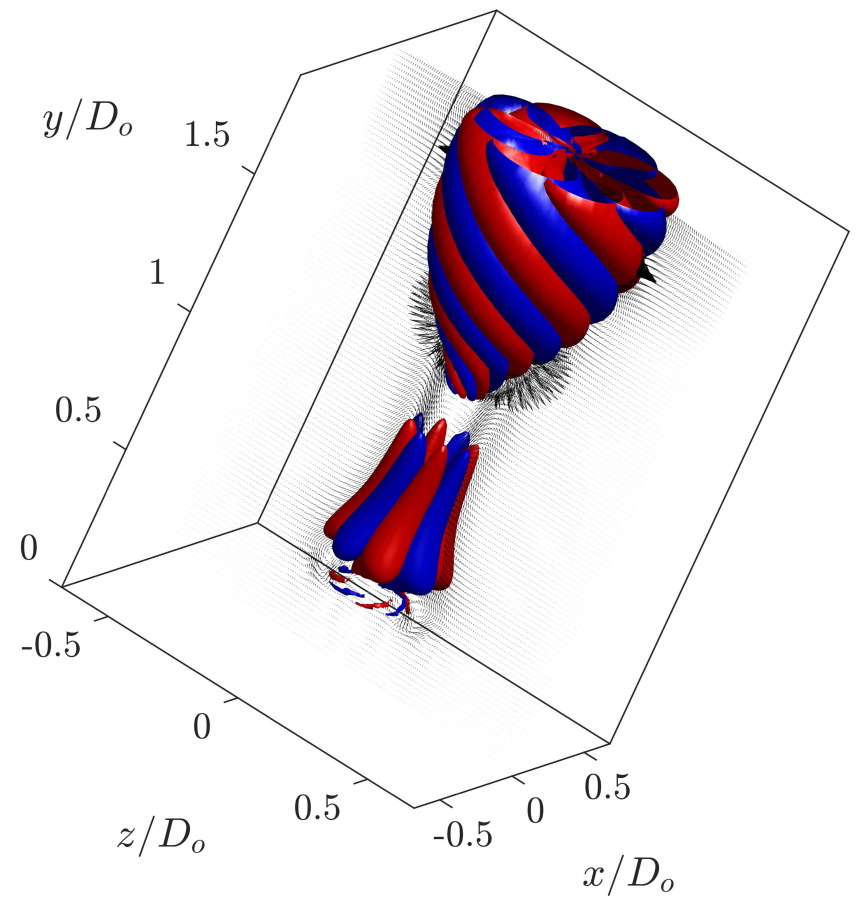

(b) Double-helical mode $(m=2)$

Figure 12: Spatial structure of the identified PVC modes obtained by global direct LSA, isosurface of $Q$ criterion (arbitrary scale and arbitrary phase angle), blue: real part, red: imaginary part; vector field shows real part of the coherent fluctuations projected onto the plane $x=0$. 


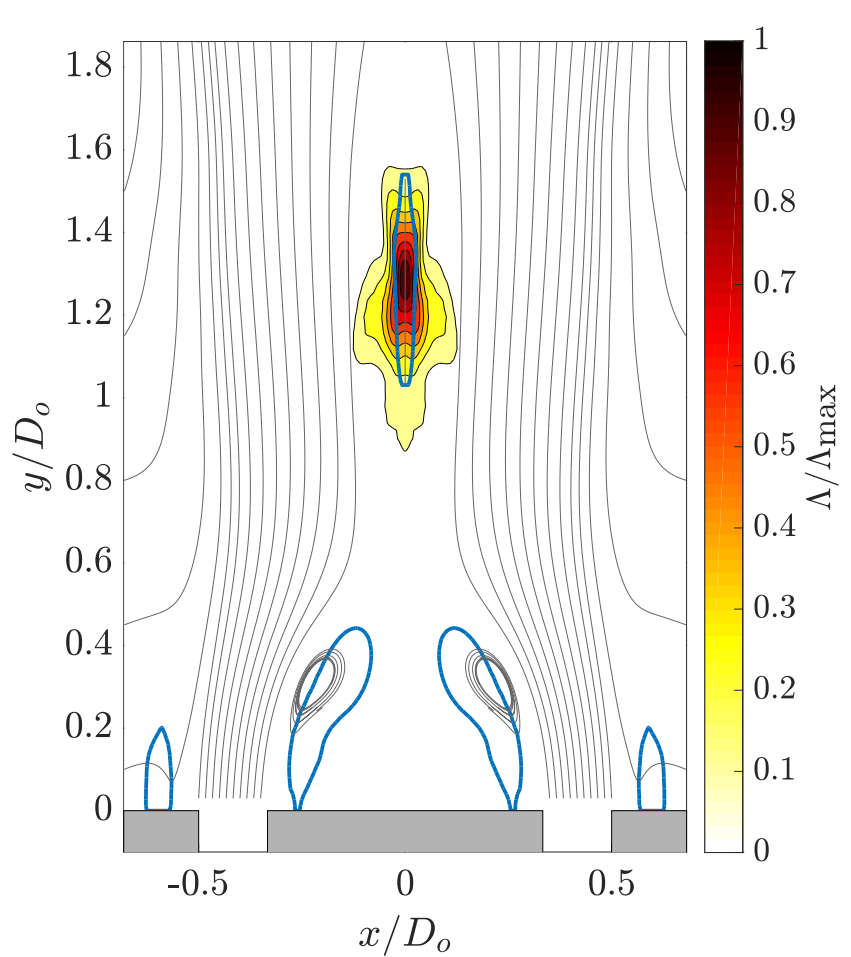

(a) Single-helical mode $(m=1)$

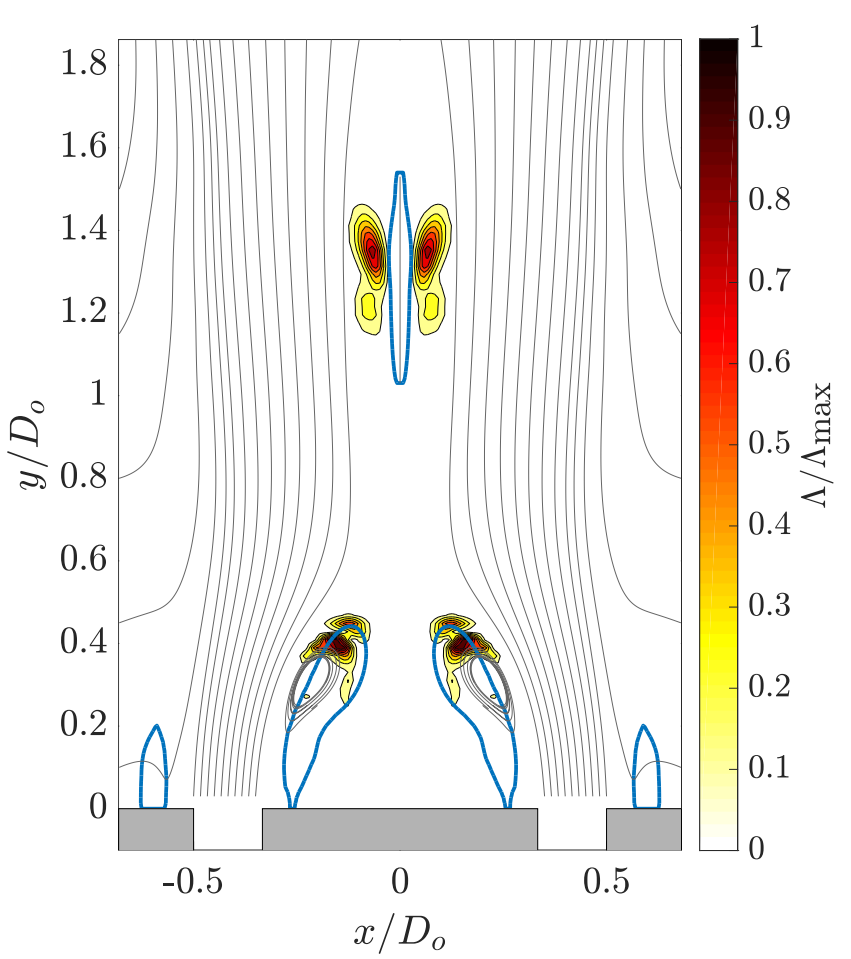

(b) Double-helical mode $(m=2)$

Figure 13: Structural sensitivity $\Lambda$ of the identified global modes obtained by global direct and adjoint LSA, grey lines denote streamlines, thick blue lines denote contours of zero axial velocity indicating the recirculation zones

well. In the second region of convective amplification, the axial wavelength is slightly underpredicted compared to the SPOD mode, leading to a steeper inclination angle of the counter-winding vortices. Furthermore, the convective growth is overpredicted, leading to a larger radial spread of the mode. The mismatch towards the outlet boundary is likely to have the same cause as the mismatch of the frequency, which is rooted in potential inaccuracies in the wavemaker region due to very strong mean flow gradients.

\subsection{Structural sensitivity and wavemaker}

Figure 13 shows the structural sensitivity of the two identified global modes. It is high where the receptivity, represented by the adjoint mode, and the forcing, represented by the direct mode, have their combined maximum. These regions of high structural sensitivity correspond to the wavemaker of the respective modes where the self-excitation mechanism acts. In both cases the wavemaker positions are associated with regions of mean recirculation, supporting a self-excited feedback loop due to reverse flow. The wavemaker of the single-helical mode is located in the center of the vortex breakdown bubble around $y / D_{o} \approx 1.3$. This thin recirculation strip, associated with the single-helix's wavemaker, is completely detached from the upstream large recirculation bubble located at the exit of the annulus. For the double-helical mode, two wavemakers exist. The first is located at the downstream end of the recirculation bubble around $y / D_{o} \approx 0.4$ and is responsible for the initial growth of the amplitude as observed in figure 12. The second wavemaker is bordered at the vortex breakdown bubble, close to the wavemaker of the single-helical mode. It is responsible for the re-initiation of growth towards the downstream end of the domain. The first, upstream lying wavemaker is the primary wavemaker of the mode since the truncation of the second, downstream lying wavemaker does not affect the frequency and the growth rate of the double-helical mode, as discussed above (also see Appendix C). The clear spatial separation of the single-helical wavemaker and the primary double-helical wavemaker demonstrates the independence of the self-excitation mechanisms. The double-helix is explicitly no higher harmonic of the single-helix and both modes have completely different sources. Moreover, the close proximity of the single-helical wavemaker and the secondary double-helical wavemaker coincides with the competition of both modes as observed for the temporal coefficients in figure 6 and 7. Notably, the bulk production of coherent kinetic energy (see figure 10) for both modes is in slight spatial shift with the single-helical and secondary double-helical wavemaker. The maximum of production is reached slightly upstream of these wavemakers. This reflects an essential feature of both global modes. Coherent fluctuations are produced and subsequently advected downstream where they are fed to their respective wavemakers, sustaining the loop of self-excitation. Surprisingly, however, this does not apply to the primary wavemaker of the double-helix. There, the main production starts downstream of the wavemaker. 


\section{Conclusions}

In this paper, the flow field of an annular swirling jet has been studied by means of time-resolved tomographic particle image velocimetry. The large-scale dynamics were extracted by classical and spectral POD. Despite the simple geometrical configuration of the annular jet, the flow field is very complex. Two distinct helical structures were found: a single-helical structure with an azimuthal wavenumber $m=1$ and a double helical structure with an azimuthal wavenumber $m=2$. The energy content of these modes was very low and both structures were only intermittently present. For this reason, classic snapshot POD failed to extract these modes in a consistent way as it distributes their dynamics across several modes. SPOD, however, delivers a mode space that allows the dynamics of the two modes to be clearly identified. The first helical structure (the single helix mode) was precessing at a frequency of $S t=0.273$, while the double helix at $S t=0.536$. Analysis of the temporal coefficients obtained by SPOD showed that both structures are no harmonics of each other. Both structures have a significant impact on the turbulent statistics and the mean flow. The calculation of coherent production shows that the single helix extracts its energy from the shear layer between the vortex breakdown bubble and the jet, while the double helix extracts a part of the energy immediately downstream of the CRZ and in the shear layer between breakdown bubble and jet.

Global linear stability analysis was employed based on the measured mean flow. Two converged global modes were identified that matched very well with the empirically found structures in terms of frequency and mode shape. Furthermore, the structural sensitivity of the two modes was calculated using the corresponding adjoint modes and the wavemaker regions were identified. The clear spatial separation of both (primary) wavemaker regions demonstrates the independence of the respective self-excitation mechanisms. This further supports that the double-helical mode is not a higher harmonic of the single-helix mode and that both modes have completely different sources. Furthermore, both modes were found to be stable but close to the stability limit, which suggests that they are only present in the data due to a continuous stochastic forcing. This is also supported by the observation that both modes are competing and each occurs only intermittently. The reason why mean flow analysis still captures both modes well is likely because both are still close to their bifurcation point and the stochastic forcing allows both to arise intermittently. To the authors' knowledge, the capability of mean flow stability analysis to capture two intermittently co-existing helical global modes in a highly three-dimensional turbulent flow has not been demonstrated before. Moreover, this paper shows that a detailed insight into the dynamics of these competing modes is only possible through the combination of the results from empirical mode identification from experimental data and analytic mode predictions from mean flow global linear stability analysis.

The corresponding author wishes to thank the FWO Flanders and the J.M. Burgerscentrum for fluid mechanics for financially supporting his stay to perform the measurements at the water tunnel facility at Delft University of Technology (TU Delft). The German Research Foundation is also acknowledged for funding this work within the project OB 402/4-3.

\section{A Considerations on accuracy of the global LSA}

Figure 14 shows, for $x / D_{o} \geq 0$, the solid body rotation rate $\bar{w} / x$ at every location in the field as well as the locations of both wavemakers. Accounting for the the single- and double-helicity of both modes, the phase rotation rate $\Omega_{m}=m \bar{w} / x$ at the wavemaker locations can be specified with $\Omega_{1} \approx 115 \mathrm{rad} / \mathrm{s}$ for $m=1$ and $\Omega_{2} \approx 220 \mathrm{rad} / \mathrm{s}$ for $m=2$. The ratio of phase rotation rate and the global frequency predicted by LSA is $\Omega_{m} /\left(2 \pi f_{\mathrm{LSA}, m}\right) \approx 0.7$ for both modes. This demonstrates that the solid body rotation rate at the wavemaker location and the global LSA frequency are closely related.

In regions where the swirling jet rotates as a solid body, the solid body rotation rate $\bar{w} / x$ should ideally stay constant with increasing distance to the centerline. In fact, the wavemaker for the single-helical mode $m=1$ is located in a region where the solid body rotation rate stays almost constant for increasing $x$. In contrast, the wavemaker for the double-helical mode $m=2$ resides in a region where the flow clearly does not rotate as a solid body since the solid body rotation rate shows a steep decrease with increasing $x$. This implies a higher sensitivity of the global LSA frequency to errors in the prediction of the wavemaker location. Since the wavemaker location is strongly related to the border of the recirculation bubble (e.g. Qadri et al. (2013)), a slight misprediction of the bubble position and thus, the wavemaker location, can have a large effect onto the prediction of the LSA frequency.

The sensitivity of the predicted LSA frequency due to uncertainties of the breakdown bubble position can be examined by, e.g., considering the vector spacing of the post-processed tomographic PIV data (see section 2.2). The vector spacing is $\delta / D_{o} \approx 0.02$. Assuming the ratio of phase rotation rate and global LSA frequency $\Omega_{m} /\left(2 \pi f_{\mathrm{LSA}, m}\right) \approx 0.7$ to be constant for slight displacements of the breakdown bubble and, thus, the wavemaker, the impact on the phase rotation rate and, thus, the predicted global LSA frequency can be estimated. For $m=1$, displacements of one unit of vector spacing $\delta / D_{o} \approx 0.02$ in $x$ - or $y$-direction would lead to a change of 


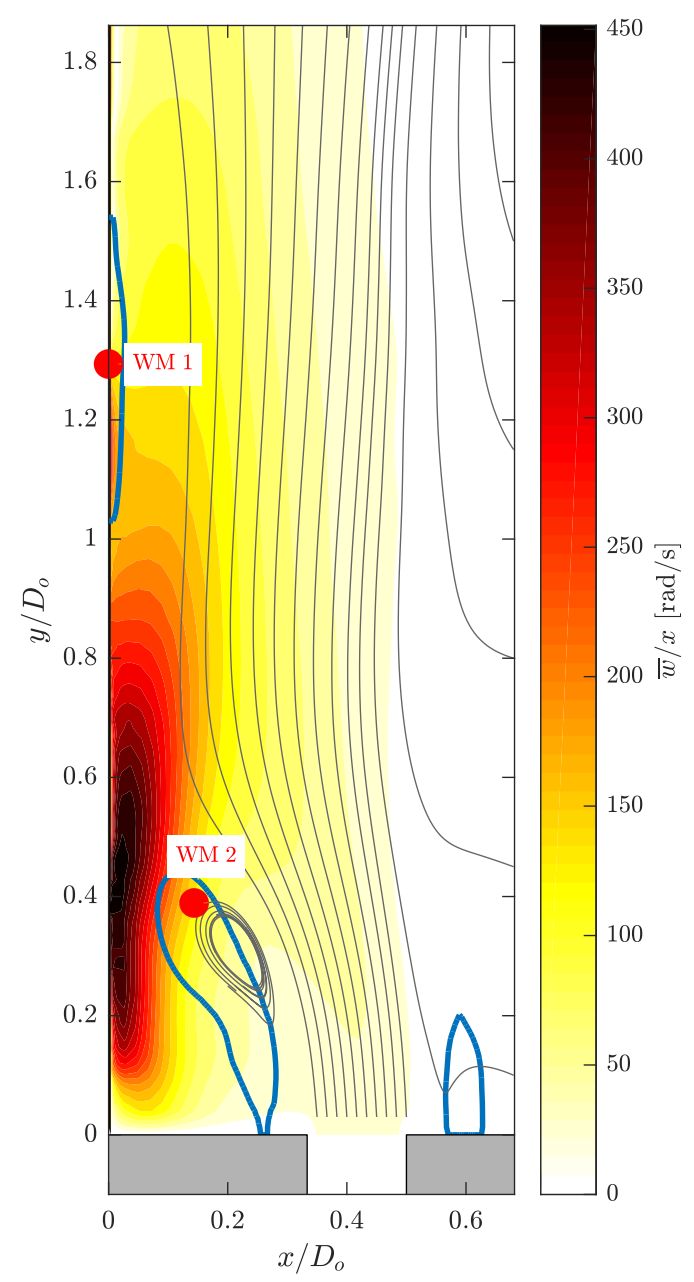

Figure 14: Mean azimuthal velocity with wavemakers for modes $m=1$ and $m=2$ corresponding to regions 1 and 2 of solid body rotation are denoted by WM 1 and WM 2, respectively; solid and dashed lines define aforementioned regions of solid body rotation

LSA frequency below $3 \%$. In contrast, a displacement of one unit of vector spacing in $y$-direction would lead to a change of LSA frequency by $4 \%$ and in $x$-direction to a change by $21 \%$. On the other hand, the relative error in comparison to the 'correct' experimentally measured global frequency is $13 \%$. Therefore, the error of the LSA frequency is actually smaller than the frequency uncertainty related to the relatively coarse vector spacing.

\section{B Considerations on convergence of the global LSA}

The main issue regarding full convergence of the selected modes of interest is the limited spatial resolution of the tomographic PIV data. In cases where the mesh grid of the global LSA is coarser than the grid of the PIV, the change of most of the eigenvalues with increasing mesh resolution follows a monotonous trend. When the mesh of the global LSA is finer than the grid of the PIV, the changes of the eigenvalues become small but seemingly 'random' since the addition of nodes does not provide any additional information anymore. These 'random' changes can be basically attributed to discretization errors caused by the employed interpolation scheme coupled with the choice of finite difference scheme. Therefore, the classical way of showing convergence of the spurious modes and of the modes of interest is difficult.

For these reasons, we resort to other means of selecting the best fitting eigenvalue to obtain the mode of interest for $m=1$ and $m=2$. Within the constraints of the modal basis, the goal is to find the eigenvalue that represents the global modes $m=1$ and $m=2$ optimally, based on inspection of 1) stability, 2) frequency and 3) mesh sensitivity. Mesh sensitivity of an eigenvalue can be used as an indicator for spurious and nonphysical modes that can be ignored in the context of global instabilities (Sipp et al., 2010). The mesh sensitivity is quantitatively assessed by calculating the standard deviation of the eigenvalue change over all considered mesh sizes. Figure 15(a) shows the eigenvalue spectrum for different mesh resolutions for $m=1$. The selected mode of interest directly fulfills criterion 1 and 2 in that it is the least stable eigenvalue while matching the measured frequency with least error. Additionally, it is one of the eigenvalues with the lowest mesh sensitivity (criterion 3 ). 


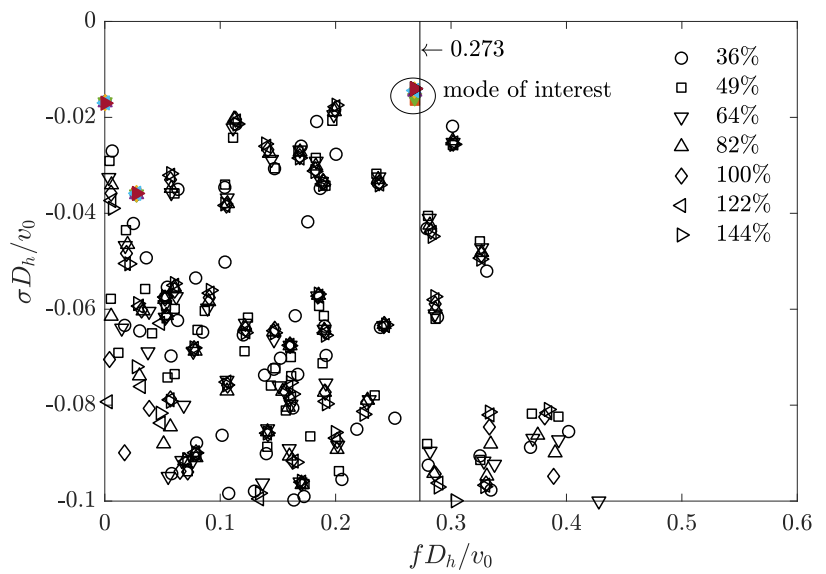

(a) $m=1$

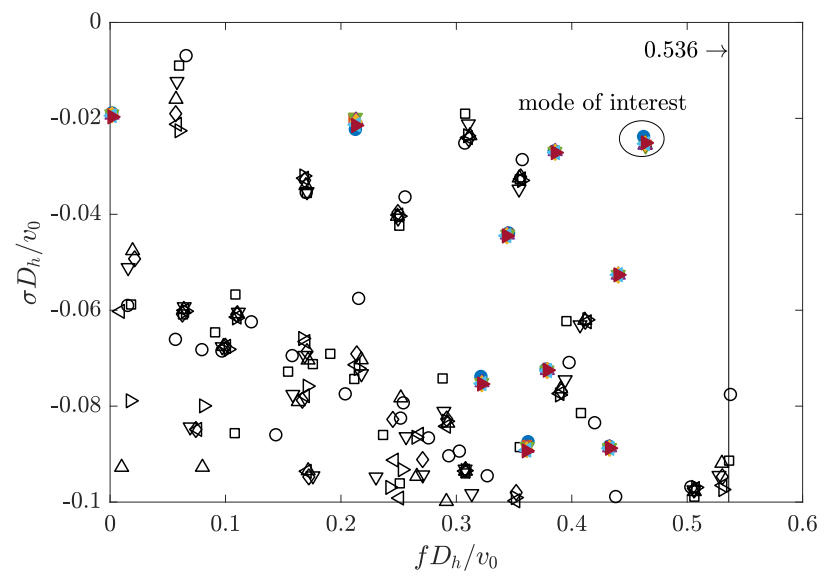

(b) $m=2$

Figure 15: Eigenvalue spectrum for different mesh resolutions, where $100 \%$ mesh resolution corresponds to reference mesh with resolution approximately equal to PIV mesh, encircled eigenvalues correspond to selected mode of interest, colored markers denote eigenvalues that are less or equally mesh-sensitive as the selected mode of interest (quantified via standard deviation), vertical line denotes experimentally measured Strouhal number

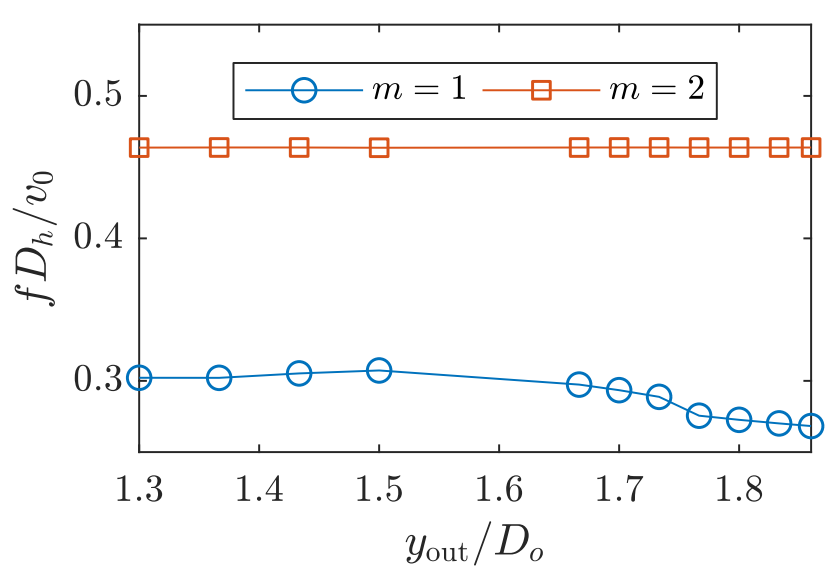

(a) Global LSA frequency $f D_{h} / v_{0}$

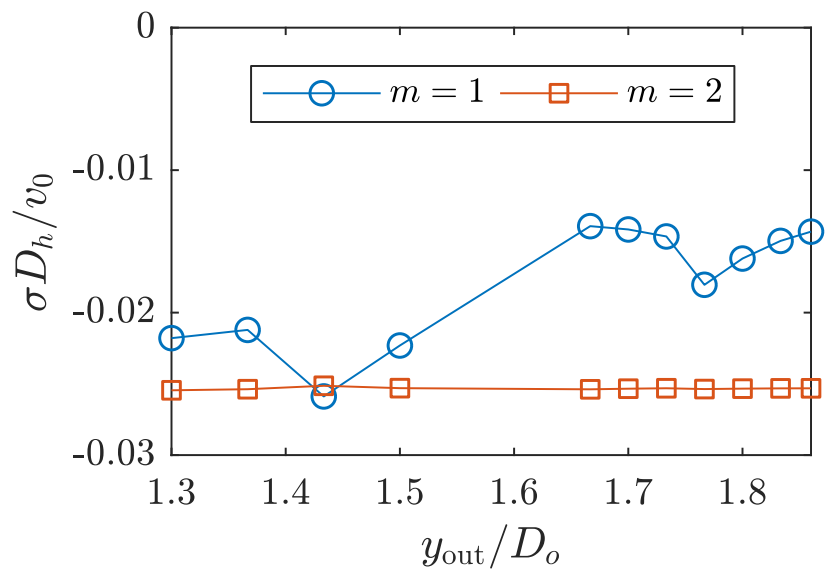

(b) Growth rate $\sigma D_{h} / v_{0}$

Figure 16: Impact of domain truncation on global LSA, $y_{\text {out }} / D_{o}$ denotes axial position of truncation where furthest to the right corresponds to no truncation and furthest to the left corresponds to more than $30 \%$ truncation of downstream domain

For $m=2$ the proper selection is more difficult (see Fig. 15(b)). The selected mode of interest is not the least stable. However, it is one of the least stable modes (criterion 1) which are still close enough to the measured frequency (criterion 2). Additionally, the eigenvalue is one of the least mesh-sensitive (criterion 3).

\section{Impact of domain truncation on global LSA}

Figure 16 shows the impact of domain truncation on the predicted global LSA frequency and growth rate. For the single-helical mode $m=1$, the predicted frequency increases by almost $13 \%$ when $30 \%$ of the downstream domain is truncated. Likewise, the growth rate shows a general trend of decrease by around $50 \%$. This demonstrates that truncating regions of the wavemaker of $m=1$, which is centered at $y / D_{o}=1.3$, affects the predictive quality of the global LSA for the single-helical mode. In contrast, the double-helical mode $m=2$ shows no sensitivity with regard to the considered domain truncations. Global LSA frequency and growth rate stay nearly constant. Although the structural sensitivity of $m=2$ is high in two isolated regions around $y / D_{o}=0.4$ and $y / D_{o}=1.3$, the insensitivity of frequency and growth rate with regard to truncating up to $y / D_{o}=1.3$ clearly demonstrates that the primary wavemaker of $m=2$ is located in the upstream region at $y / D_{o}=0.4$.

\section{References}

AtKinson, C. \& Soria, J. 2009 An efficient simultaneous reconstruction technique for tomographic particle 
image velocimetry. Exp. Fluids 47, 553.

BARKLEY, D. 2006 Linear analysis of the cylinder wake mean flow. Europhys. Lett. 75, 750-756.

BeÉr, J. \& Chigier, N. 1983 Combustion Aerodynamics. Krieger Pub Co.

Benjamin, T. B. 1962 Theory of the vortex breakdown phenomenon. Journal of Fluid Mechanics 14 (4), $593-629$.

Billant, P., Chomaz, J.-M. \& Huerre, P. 1998 Experimental study of vortex breakdown in swirling jets. Journal of Fluid Mechanics 376, 183-219.

BorEÉ, J. 2003 Extended proper orthogonal decomposition: a tool to analyse correlated events in turbulent flows. Experiments in Fluids 35, 188-192.

Brackston, R.D., de la Cruz, J.M. Garca, Wynn, A., Rigas, G. \& Morrison, J. F. 2016 Stochastic modelling and feedback control of bistability in a turbulent bluff body wake. Journal of Fluid Mechanics $\mathbf{8 0 2}$, $726-749$

Calderon, D. E., Wang, Z. \& Gursul, I. 2012 Three-dimensional measurements of vortex breakdown. Experiments in Fluids 53, 293-299.

Canepa, E., Cattanei, A., Lengani, D., Ubaldi, M. \& Zunino, P. 2015 Experimental investigation of the vortex breakdown in a lean premixing prevaporizing burner. Journal of Fluid Mechanics $\mathbf{7 6 8}$ (R4).

Chomaz, JeAn-Marc 2005 Global instabilities in spatially developing flows: non-normality and nonlinearity. Annu. Rev. Fluid Mech. 37, 357-392.

Crouch, J.D., Garbaruk, A. \& Magidov, D. 2007 Predicting the onset of flow unsteadiness based on global instability. Journal of Computational Physics 224 (2), 924 - 940.

Crouch, J.D., Garbaruk, A., Magidov, D. \& Travin, A. 2009 Origin of transonic buffet on aerofoils. Journal of Fluid Mechanics 628, 357-369.

Danlos, A. \& Patte-Rouland, B. 2013 Experimental characterization of the initial zone of an annular jet with a very large diameter ratio. Experiments in Fluids 54, 1418.

Dinesh, K.K.J. Range, Jenkins, K.W., Kirkpatrick, M.P. \& Malalasekera, W. 2012 Effects of swirl on intermittency characteristics in non-premixed flames. Combustion Science and Technology 184 (5), 629-659.

Dinesh, K.K.J. RANGe \& KiRKPATRICK, M.P. 2009 Study of jet precession, recirculation and vortex breakdown in turbulent swirling jets using les. Computers and Fluids 38, 1232-1242.

Dugué, J. \& WeBer, R. 1992 Design and calibration of a 30kw natural gas burner for the university of michigan. Tech. Rep. Doc. No. C74/y/1. IJmuiden, The Netherlands.

Durao, D.F.G. \& Whitelaw, J.H. 1978 Velocity characteristics of the flow in the near wake of a disk. Journal of Fluid Mechanics 85, 369-385.

Escudier, M. P. \& Zehnder, N. 1982 Vortex-flow regimes. Journal of Fluid Mechanics 115, $105-121$.

Falese, M., Gicquel, L.Y. \& Poinsot, T. 2014 Les of bifurcation and hysteresis in confined annular swirling flows. Computers and Fluids 89, 167-178.

Gallaire, F., Rott, S. \& Chomaz, J.-M. 2004 Experimental study of a free and forced swirling jet. Physics of Fluids 16, 2907-2917.

Gallaire, F., Ruith, M., Meiburg, E., Chomaz, J.-M. \& Huerre, P. $2006 a$ Spiral vortex breakdown as a global mode. Journal of Fluid Mechanics 549, 71-80.

Gallaire, Francois, Ruith, Michael, Meiburg, Eckart, Chomaz, Jean-Marc \& Huerre, Patrick $2006 b$ Spiral vortex breakdown as a global mode. Journal of Fluid Mechanics 549, 71-80.

Garcia-Villalba, M. \& Frohlich, J. 2006 Les of a free annular swirling jet - dependence of coherent structures on a pilot jet and the level of swirl. International Journal of Heat and Fluid Flow 27, 911-923.

Garcia-Villalba, M., Frohlich, J. \& Rodi, W. 2006 Identification and analysis of coherent structures in the near field of a turbulent unconfined annular swirling jet using large eddy simulation. Physics of Fluids $\mathbf{1 8}$ 055103 . 
Gentille, V., van Oudheusden, B.W., Schrijer, F.F.J. \& Scarano, F. 2017 The effect of angular misalignment on low-frequency axisymmetric wake instability. Journal of Fluid Mechanics 813 (R3).

Gentille, V., Schrijer, F.F.J., van Oudheusden, B.W. \& Scarano, F. 2016 Low-frequency behavior of the turbulent axisymmetric near-wake. Physics of Fluids 28 (065102).

Giannetti, Flavio \& Luchini, Paolo 2007 Structural sensitivity of the first instability of the cylinder wake. Journal of Fluid Mechanics 581, 167-197.

Grandemange, M., Gohlke, M. \& Cadot, O. 2013 Turbulent wake past a three-dimensional blunt body. part 1. global modes and bi-stability. Journal of Fluid Mechanics 722, 51-84.

Gupta, A. \& Lilley, G. 1984 Swirl Flows. Abacus, Kent.

Huang, Y., ans S.Y. Hsieh, H.G. Sung \& Yang, V. 2003 Large-eddy simulation of combustion dynamics of lean-premixed swirl-stabilized combustor. Journal of Propulsion and Power 19, 782-794.

HuANG, Y. \& YANG, V. 2005 Effect of swirl on combustion dynamics in a lean-premixed swirl-stabilized combustor. Proceedings of the Combustion Institute 30, 1775-1782.

Hussain, A.K.M.F. \& Reynolds, W.C. 1970 The mechanics of an organized wave in turbulent shear flow. Journal of Fluid Mechanics 41, 241-258.

Ivanova, Elizaveta M, Noll, Berthold E \& Aigner, Manfred 2013 A numerical study on the turbulent schmidt numbers in a jet in crossflow. Journal of Engineering for Gas Turbines and Power 135 (1), 011505.

Jeong, J. \& Hussain, F. 1995 On the identification of a vortex. Journal of Fluid Mechanics 285, 69-94.

Jones, W.P., Lyra, S. \& Navarro-Martinex, S. 2012 Large eddy simulation of turbulent confined highly swirling annular flows. Flow, Turbulence and Combustion 89, 361-384.

Kaiser, Thomas L, Poinsot, Thierry \& Oberleithner, Kilian 2017 Stability and sensitivity analysis of hydrodynamic instabilities in industrial swirled injection systems. In ASME Turbo Expo 201\%: Turbomachinery Technical Conference and Exposition. American Society of Mechanical Engineers.

Ko, N.W.M. \& Chan, W.T. 1979 Inner regions of annular jets. Journal of Fluid Mechanics 93, 549-584.

Lambourne, N. C. \& Bryer, D. W. 1961 The bursting of leading-edge vortices: some observations and discussion of the phenomenon. Tech. Rep. 3282. Ministry of Aviation, Aeronautical Research Council.

Liang, H. \& Maxworthy, T. 2005 An experimental investigation of swirling jets. Journal of Fluid Mechanics 525, 115-159.

Lucas, J.-M., Cadot, O., Herbert, V., Parpais, S. \& Delery, J. 2017 A numerical investigation of the asymmetric wake mode of a squareback ahmed body - effect of a base cavity. Journal of Fluid Mechanics 831 (675-697).

Lucch-Negro, O. \& O’Doherty, T. 2001 Vortex breakdown: a review. Progress in Energy and Combustion Science 27, 431-481.

Luchini, Paolo \& Bottaro, Alessandro 2014 Adjoint equations in stability analysis. Annual Review of fluid mechanics 46.

Lumley, J.L. 1970 Stochastic tools in turbulence. Applied mathematics and mechanics series: Volume 12. New York: Academic press.

Mantič-Lugo, Vladislav, Arratia, Cristóbal \& Gallaire, François 2015 A self-consistent model for the saturation dynamics of the vortex shedding around the mean flow in the unstable cylinder wake. Physics of Fluids 27 (7), 074103.

Matas, J.-P., Delon, A. \& CARtellier, A. 2018 Shear instability of an axisymmetric air-water coaxial jet. Journal of Fluid Mechanics 843, 575-600.

Meliga, P., Cadot, V \& Serre, E. 2016 Experimental and theoretical sensitivity analysis of turbulent flow past a square cylinder. Flow, turbulence and combustion 4.

Meliga, P., Gallaire, F. \& Chomaz, J.-M. $2012 a$ A weakly nonlinear mechanism for mode selection in swirling jets. Journal of Fluid Mechanics 699, 216-262. 
Meliga, P., Pujals, G. \& Serre, E. $2012 b$ Sensitivity of 2-d turbulent flow past a d-shaped cylinder using global stability. Physics of Fluids 24 (6), 061701.

Mettot, Clment, Sipp, Denis \& Bzard, Herv 2014 Quasi-laminar stability and sensitivity analyses for turbulent flows: Prediction of low-frequency unsteadiness and passive control. Physics of Fluids 26 (4), 045112.

Oberleithner, K., Paschereit, C.O., Seele, R. \& Wygnanski, I. 2012 Formation of turbulent vortex breakdown: Intermittency, criticality, and global instability. AIAA Journal 50 (7), 1437-1452.

Oberleithner, K., Sieber, M., Nayeri, C.N., Paschereit, C.O., Petz, C., Hege, H.C., Noack, B.R. \& Wygnanski, I. 2011 Three-dimensional coherent structures in a swirling jet undergoing vortex breakdown: stability analysis and empirical mode construction. Journal of Fluid Mechanics 679, 383-414.

Oberleithner, K., Stöhr, M., Seong, H.I., Arndt, C.M. \& Steinberg, A.M. 2015 Formation and flame-induced supression of the precessing vortex core in a swirl combustor: Experiments and linear stability analysis. Combustion and Flame 162 (8), 3100-3114.

O'Connor, J. \& Lieuwen, T. 2012 Recirculation zone dynamics of a transversal excited swirl flow and flame. Physics of Fluids 24, 075107.

PARedes, P 2014 Advances in global instability computations: from incompressible to hypersonic flow. PhD thesis, Technical University of Madrid, Madrid, Spain.

Patte-Rouland, B., Lalizel, G., Moreau, J. \& Rouland, E. 2001 Flow analysis of an annular jet by particle image velocimetry and proper orthogonal decomposition. Measurement Science and Technology $\mathbf{1 2}$ $1404-1412$.

Pavia, G., Passmore, M. \& SArdu, C. 2018 Evolution of the bi-stable wake of a square-back automotive shape. Experiments in Fluids $\mathbf{5 9}$ (20).

Percin, M., Vanierschot, M. \& van Oudheusden, B.W. 2017 Analysis of the pressure fields in a swirling annular jet flow. Experiments in Fluids 58 (166).

Poinsot, T. 2017 Prediction and control of combustion instabilities in real engines. Proceedings of the Combustion Institute 36, 1-28.

QAdri, U. A., Mistry, D. \& Juniper, M. P. 2013 Structural sensitivity of spiral vortex breakdown. Journal of Fluid Mechanics $\mathbf{7 2 0}, 558-581$.

Reynolds, WC \& Hussain, AKMF 1972 The mechanics of an organized wave in turbulent shear flow. part 3. theoretical models and comparisons with experiments. Journal of Fluid Mechanics 54 (2), 263-288.

Rigas, G., Morgans, A.S., Brackston, R.D. \& Morrison, J.F. 2015 Diffusive dynamics and stochastic models of turbulent axisymmetric wakes. Journal of Fluid Mechanics 778, R2.

Rigas, G., Oxlade, A.R., Morgans, A.S. \& Morrison, J.F. 2014 Low-dimensional dynamics of a turbulent axisymmetric wake. Journal of Fluid Mechanics 755, R5.

Rowley, C.W., Mezić, I., Bagheri, S., Schlatter, P. \& Henningson, D.S. 2009 Spectral analysis of nonlinear flows. Journal of Fluid Mechanics 641, 115-127.

Ruith, M., Chen, P., Meiburg, E. \& Maxworthy, T. 2003 Three-dimensional vortex breakdown in swirling jets and wakes. Journal of Fluid Mechanics 486, 331-378.

Rukes, Lothar, Paschereit, Christian Oliver \& Oberleithner, Kilian $2016 a$ An assessment of turbulence models for linear hydrodynamic stability analysis of strongly swirling jets. European Journal of MechanicsB/Fluids 59, 205-218.

Rukes, L., Paschereit Oliver, C. \& Oberleithner, K. $2016 b$ An assessment of turbulence models for linear hydrodynamic stability analysis of strongly swirling jets. European Journal of Mechanics - B/Fluids $\mathbf{5 9}$, $205-218$.

Rukes, L., Sieber, M., Paschereit, C.O. \& Oberleithner, K. 2017 Transient evolution of the global mode in turbulent swirling jets: Experiments and modal stability analysis. European Journal of Mechanics B/Fluids 65, 98-106.

SARPKAYA, T. 1971 On stationary and travelling vortex breakdowns. Journal of Fluid Mechanics 45, 545-559. 
Schmid, P.J. 2010 Dynamic mode decomposition of numerical and experimental data. Journal of Fluid Mechanics 656, 5-28.

Sheen, H, Chen, W \& Jeng, S 1996 Recirculation zones of unconfined and confined annular swirling jets. AIAA Journal 34, 572-579.

Sieber, M., Paschereit, C. O. \& Oberleithner, K. 2016 Spectral proper orthogonal decomposition. Journal of Fluid Mechanics 792, 798-828.

Sipp, Denis, Marquet, Olivier, Meliga, Philippe \& Barbagallo, Alexandre 2010 Dynamics and control of global instabilities in open-flows: a linearized approach. Applied Mechanics Reviews 63 (3), 030801.

Sirovich, L. 1987 Turbulence and the dynamics of coherent structures. part i: Coherent structures. Quaterly of Applied Mathematics 45, 561-571.

Stoenr, M., Boxx, I. \& Campbell, C. D. 2012 Experimental study of vortex-flame interaction in a gas turbine model combustor. Combustion and Flame 159, 2636-2649.

SyRED, N. 2006 A review of oscillation mechanisms and the role of the precessing vortex core (pvc) in swirl combustion systems. Progress in Energy and Combustion Science 32 (2), 93-161.

TAmmisola, O. \& Juniper, M.P. 2016 Coherent structures in a swirl injector at re $=4800$ by nonlinear simulations and linear global modes. Journal of Fluid Mechanics 792, 620-657.

Terhaar, S., Oberleithner, K. \& Paschereit, C.O. 2015 Key parameters governing the precessing vortex core in reacting flows: An experimental and analytical study. Proceedings of the Combustion Institute 35 (3), $3347-3354$.

Theofilis, V. 2011 Global Linear Instability. Annu. Rev. Fluid Mech. 43, 319-352.

Theofilis, V, Duck, PW \& Owen, J 2004 Viscous linear stability analysis of rectangular duct and cavity flows. Journal of Fluid Mechanics 505, 249-286.

Turton, Sam E, Tuckerman, Laurette S \& Barkley, Dwight 2015 Prediction of frequencies in thermosolutal convection from mean flows. Physical Review E 91 (4), 043009.

Vadivukkarasan, M. \& Panchagnula, M.V. 2017 Combined rayleigh-taylor and kelvin-helmholtz instabilities on an annular liquid sheet. Journal of Fluid Mechanics 812, 152-177.

VANIERSCHOT, M. 2017 On the dynamics of the transition to vortex breakdown in axisymmetric inviscid swirling flows. European Journal of Mechanics B/Fluids 65, 65-69.

Vanierschot, M., Percin, M. \& van Oudheusden, B.W. 2018 Double helix vortex breakdown in a turbulent swirling annular jet flow. Physical Review Fluids 3 (034703).

VAnierschot, M. \& VAN DEN BulcK, E. 2008 Influence of swirl on the initial merging zone of a turbulent annular jet. Physics of Fluids 20, 105104.

VAnierschot, M. \& VAn Den Bulck, E. 2011 Experimental study of low precessing frequencies in the wake of a turbulent annular jet. Experiments in Fluids 50, 189-200.

Vanierschot, M., Van Dyck, K., Sas, P. \& VAn Den Bulck, E. 2014 Symmetry breaking and vortex precession in low-swirling annular jets. Physics of Fluids 26, 105110.

WiEnEKE, B. 2008 Volume self-calibration for 3d particle image velocimetry. Exp. Fluids 45, 549556.

Williams, M.O., Kevrekidis, I.G. \& Rowley, C.W. 2015 A data-driven approximation of the koopman operator: Extending dynamic mode decomposition. Journal of Nonlinear Science 25 (1307-1346).

Zhang, H.D., Han, C., Ye, T.H., Zhang, J.M. \& Chen, Y. 2015 Large eddy simulation of unconfined turbulent swirling flow. Science China Technological Sciences 58, 1731-1744. 